\title{
Slow coevolution of a viral pathogen and its diploid host
}

Andreasen, Viggo; Christiansen, Freddy B.

\author{
Publication date:
}

1994

\section{Document Version}

Publisher's PDF, also known as Version of record

Citation for published version (APA):

Andreasen, V., \& Christiansen, F. B. (1994). Slow coevolution of a viral pathogen and its diploid host. Roskilde Universitet. Tekster fra IMFUFA No. 285 http://milne.ruc.dk/ImfufaTekster/

\section{General rights}

Copyright and moral rights for the publications made accessible in the public portal are retained by the authors and/or other copyright owners and it is a condition of accessing publications that users recognise and abide by the legal requirements associated with these rights.

- Users may download and print one copy of any publication from the public portal for the purpose of private study or research. - You may not further distribute the material or use it for any profit-making activity or commercial gain.

- You may freely distribute the URL identifying the publication in the public portal.

\section{Take down policy}

If you believe that this document breaches copyright please contact rucforsk@kb.dk providing details, and we will remove access to the work immediately and investigate your claim. 


\section{TEKST NR 285}

1994

Slow coevolution of a viral

pathogen and its diploid host

Viggo Andreasen and Freddy B. Christiansen

TEKSTER fra

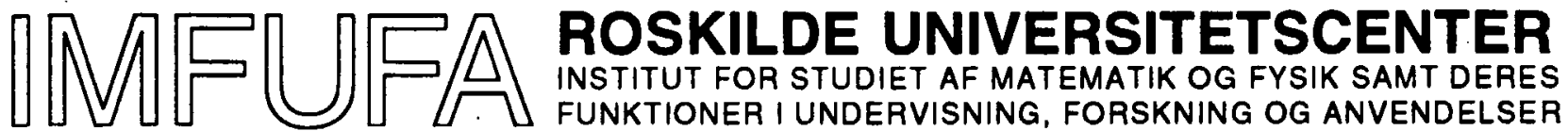


IMFUFA, Roskilde Universitetscenter, Postboks 260, 4000 Roskilde

Slow coevolution of a viral pathogen and its diploid host ${ }^{1}$

by: Viggo Andreasen and Freddy B. Christiansen

IMFUFA tekst nr. 285/94 30 pages

ISSN 0106-6242

\begin{abstract}
We study a population exposed to a lethal infectious disease. Host response is carried at one locus with two alleles while the pathogen occurs in two variants. Based on an SI-type epidemic model we derive explicit equations for the dynamics of each genotype. By assuming small variations in both host and disease, we obtain a separation in time scales between epidemic and evolutionary processes. This allows us to explicitly describe the changes in host and disease gene frequencies. The resulting model has a rich behavior including multiple stable states and oscillations. However, in the oscillatory situation the model is degenerate excluding the possibility of limit cycles. We show that the degeneracy can only be removed by frequency dependent selection in the pathogen, for example by including direct interaction of virus in a free-living stage.
\end{abstract}

${ }^{1}$ Supported in part by grant 11-9639-1 from the Danish Natural Science Research Council. 


\section{Slow coevolution of a viral pathogen and its diploid host}

\section{Introduction}

Infectious parasites and their hosts are expected to coevolve tightly due to the major impact they exert on each others reproduction and survival (Ewald, 1983). The properties of parasites and hosts may evolve in an arms race, presumably with the speed of the red queen, or they may evolve towards benign coexistence. The dynamics of genetic variation in hosts or parasites is affected by the interaction, and host-parasite interactions may be a cause for the maintenance of genetic polymorphism (Clarke, 1979).

Starting with the work of Haldane (1949) much theoretical attention has been given to host-parasite coevolution. The early work generally focused on the gene-for-gene systems described in cereal crops and their pests (see Levin, 1983 for a review). Later, models addressing more general phenomena of host-parasite coevolution have appeared (Jayakar, 1970; Yu, 1972; Lewis, 1981). These models utilize the population genetic framework of Wright (1955) and focus on the dynamics of gene frequencies. Little attention is paid to the density dependent-effects of the epidemic interactions (Levin and Udovic, 1977).

The interaction of host genetics and epidemiology was first studied by Gillespie (1975). A continuous time epidemiological model was used to derive expressions for genotypic fitnesses, and these were applied in a discrete time model of the change in host gene frequency. Kemper (1982), Longini (1983), and May and Anderson (1983) further developed this approach.

The population genetics of viral pathogens has been modeled in the frame of SIR-type models for the disease transmission dynamics. These models view the number of hosts infected by each viral type as an expression of the number of virus particles of each type. The basic unit of selection therefore becomes the viral subpopulation of each infected host individual, and viral reproduction becomes identical to infection of new hosts (Levin and Pimentel, 1981). The approach ignores viral variation and the possibility of selection within the individual host as caused by mutation or superinfection although the dynamics within the individual host seems to be important (Knolle, 1989; Sasaki and Iwasa, 1991; Nowak and May, 1994). With density independent mortality and simple transmission only the viral type with the highest transmission potential will persist in the population (Levin and Pimentel, 1981; Anderson and May, 1982; Bremermann and Thieme, 1989). The transmission potential is given by

$$
R_{0}=\frac{\beta N}{\mu+\nu}
$$


where $N$ is the population size, $\mu$ is the mortality rate, $\nu$ is the rate at which infectious hosts cease to infect due to causes other than non-disease related mortality $\mu$, and $\beta$ is the transmission coefficient.

Due to the complexity of the problem, few models of coevolution based on epidemiologically justified assumptions have appeared (May and Anderson, 1983). We will analyze a simple SIR-type coevolutionary model and follow the formulation of Beck (1984). The dynamics of each genotype of the host in each disease class and type will be described explicitly, and still following Beck, we then simplify the analysis by assuming the the variation among types is small in both host and pathogen and that the hosts reproduce by random mating. This leads to the classical weak selection approximation of the evolution of the system (Norton, 1928; Fisher, 1930; Kimura, 1958; Nagylaki, 1976), in that small differences in dynamical parameters leads to small fitness differences among host genotypes and between viral types. The assumption of small differences in fitness implies that the system will move to a quasi steady state characterized by epidemic equilibrium and Hardy-Weinberg proportions among genotypes in the host population. The dynamics of the gene frequencies in host and pathogen along this quasi steady state will be described by the small fitness differences determined from the dynamical parameters of the model. The assumption of small variation essentially linearizes the problem, and so we can exclude coexistence of viral types and host polymorphism mediated by density dependent effects.

Beck et al. (1984) found the slow dynamics along the quasi steady state by a rather complicated perturbation technique in the case of genetic variation in the host only. Recently, we have obtained the same result by simpler means (Andreasen and Christiansen, 1993). The system of Beck and coworkers was transformed into new variables that focus on the population genetic description af each disease class, and we develop this approach in the present analysis of co-evolution of host and pathogen. The ensuing model of slowly coevolving host and pathogen turns out to be degenerate in a way that prohibits the appearance of limit cycles in the genetic composition of the populations. The basic assumptions about disease transmission cause this degeneracy by excluding frequency dependent selection in the haploid virus population. The degeneracy is therefore a general property of SIR-type models. Frequency dependent selection may naturally occur for vira with a free living stage, like baculo vira, and we discuss a model where the degeneracy is broken and complicated dynamics allowed.

\section{Coevolution of host and pathogen}

We consider a lethal disease with no latent period and assume that transmission is purely horizontal with no vertical transmission occurring. Following the classical epidemic models we divide the population into two classes susceptibles $\mathrm{S}$ and infectious I (Dietz, 1975; 
Hethcote, 1974). The assumption of lethality is included to simplify the presentation, and the treatment of the model is readily extended to a full SIR-model.

The number (or density) of susceptibles in the population is $S$ and that of infectious is $I$ with a total population size of $N=S+I$. We assume that the population is well mixed so the rate at which susceptibles get infected is proportional to $I$; this force of infection is therefore $\Lambda=\beta I$. This specifies the well known SI-model with variable population size:

$$
\begin{aligned}
& \dot{S}=\dot{b} N-\mu S-\beta I S \\
& \dot{I}=\beta I S-\mu I-\nu I
\end{aligned}
$$

(Anderson and May, 1979). The parameters of the model may depend on the total population size, i. e. birth rate $b=b(N)$, the mortality rate in the absence of disease $\mu=$ $\mu(N)$, the excess mortality rate due to the disease $\nu=\nu(N)$ as well as the transmission coefficient $\beta=\beta(N)$ are allowed to be density dependent.

When $b(N)$ is a decreasing function and $\mu(N)$ and $\nu(N)$ are increasing functions satisfying $\mu(\infty)>b(0)>\mu(0)$, then the population size will remain bounded by the disease free equilibrium where $S^{+}=N^{+}$and $b\left(N^{+}\right)=\mu\left(N^{+}\right)$. In most reasonable circumstances, in particular when the contact rate $\beta(N) N$ is decreasing, the system (2) has at most one endemic equilibrium $\left(S^{*}, I^{*}\right)$ where the disease is present (Pugliese, 1990). The endemic equilibrium exists when the transmission potential at $N^{+}$,

$$
R_{0}=\frac{\beta\left(N^{+}\right) N^{+}}{\mu\left(N^{+}\right)+\nu\left(N^{+}\right)},
$$

exceeds unity and when the equilibrium exists, it is always stable (Pugliese, 1990). In the rest of this paper we will assume that the parameters are chosen so this unique, stable and endemic equilibrium exists.

We now introduce into this model the genetic structure of the host and virus populations by explicitly describing the dynamics for the number of each genotype. The disease occurs in two variants $v$ and $V$, and we divide the infectives in two classes $\hat{I}$ and I comprised of individuals infected with $v$ and $V$, respectively. We denote the numbers in the two classes by $\hat{I}$ and $\check{I}$. The response of the hosts to the disease is influenced by an autosomal locus with two alleles $a$ and $A$ and the number in the S-, $\hat{\mathrm{I}}$ - and $\mathrm{I}$-classes of each of the three genotypes $a a, a A$, and $A A$ is denoted with subscript 1,2 , and 3 , respectively. Mating is random and we assume that birth is independent of disease class and genotype. The number of $a a$-births in the population per time unit becomes $B_{1}=p^{2} b N$ where $p=\left(N_{1}+\frac{1}{2} N_{2}\right) / N$ denotes the frequency of $a$. The population birth rates for the other genotypes are $B_{2}=2 p q b N$ and $B_{3}=q^{2} b N$ where $q=1-p=\left(N_{3}+\frac{1}{2} N_{2}\right) / N$ is the frequency of $A$. The birth rates may depend additively on disease class and genotype without causing serious complications (Andreasen and Christiansen, 1993), but to keep the model simple we assume no fecundity effects. 
The genetic variation is small and its magnitude will be measured by the parameter $\varepsilon$. The genotypic variation in the host is shown by subscripted parameters and the variations in viral types are marked with "and ${ }^{\circ}$. For instance, the transmission of the disease caused by virus $v$ to susceptibles of genotype $a a$ is described by the transmission coefficient $\beta+\varepsilon \hat{\beta}_{1}$. Variation in the force of infection, however, has two sources. Variation in the transmission coefficient may be understood as variation in a genotypic susceptibility factor describing the probability per unit time that infection occurs given the amount of virus present. The amount of virus particles present in the population may also vary as a function of host genotypic composition, and we may write the force of infection as $\Lambda=\beta \Theta$ where $\Theta$ measures the ammount of available virus,

$$
\hat{\Theta}=\sum_{i=1}^{3}\left(1+\varepsilon \hat{\hat{t}}_{i}\right) \hat{I}_{i} \quad \text { and } \quad \check{\Theta}=\sum_{i=1}^{3}\left(1+\varepsilon \check{\tau}_{i}\right) \check{I}_{i}
$$

in virus population $v$ and virus population $V$, respectively. Thus, we obtain the coevolution model of Beck (1984) in the form of equations for the three genotypes in the susceptible class $\mathrm{S}$,

$$
\begin{aligned}
& \frac{d S_{1}}{d t}=B_{1}-\left[\mu+\varepsilon \mu_{1}\right] S_{1}-\left[\beta+\varepsilon \hat{\beta}_{1}\right] S_{1} \hat{\Theta}-\left[\beta+\varepsilon \check{\beta}_{1}\right] S_{1} \check{\Theta} \\
& \frac{d S_{2}}{d t}=B_{2}-\left[\mu+\varepsilon \mu_{2}\right] S_{2}-\left[\beta+\varepsilon \hat{\beta}_{2}\right] S_{2} \hat{\Theta}-\left[\beta+\varepsilon \check{\beta}_{2}\right] S_{2} \check{\Theta} \\
& \frac{d S_{3}}{d t}=B_{3}-\left[\mu+\varepsilon \mu_{3}\right] S_{3}-\left[\beta+\varepsilon \hat{\beta}_{3}\right] S_{3} \hat{\Theta}-\left[\beta+\varepsilon \check{\beta}_{3}\right] S_{3} \check{\Theta}
\end{aligned}
$$

in the infectious class $\hat{\mathrm{I}}$,

$$
\begin{aligned}
& \frac{d \hat{I}_{1}}{d t}=\left[\beta+\varepsilon \hat{\beta}_{1}\right] S_{1} \hat{\Theta}-\left[\mu+\varepsilon \mu_{1}\right] \hat{I}_{1}-\left[\nu+\varepsilon \hat{\nu}_{1}\right] \hat{I}_{1} \\
& \frac{d \hat{I}_{2}}{d t}=\left[\beta+\varepsilon \hat{\beta}_{2}\right] S_{2} \hat{\Theta}-\left[\mu+\varepsilon \mu_{2}\right] \hat{I}_{2}-\left[\nu+\varepsilon \hat{\nu}_{2}\right] \hat{I}_{2} \\
& \frac{d \hat{I}_{3}}{d t}=\left[\beta+\varepsilon \hat{\beta}_{3}\right] S_{3} \hat{\Theta}-\left[\mu+\varepsilon \mu_{3}\right] \hat{I}_{3}-\left[\nu+\varepsilon \hat{\nu}_{3}\right] \hat{I}_{3}
\end{aligned}
$$

and in the infectious class $\check{I}$,

$$
\begin{aligned}
& \frac{d \check{I}_{1}}{d t}=\left[\beta+\varepsilon \check{\beta}_{1}\right] S_{1} \check{\Theta}-\left[\mu+\varepsilon \mu_{1}\right] \check{I}_{1}-\left[\nu+\varepsilon \check{\nu}_{1}\right] \check{I}_{1} \\
& \frac{d \check{I}_{2}}{d t}=\left[\beta+\varepsilon \check{\beta}_{2}\right] S_{2} \check{\Theta}-\left[\mu+\varepsilon \mu_{2}\right] \check{I}_{2}-\left[\nu+\varepsilon \check{\nu}_{2}\right] \check{I}_{2} \\
& \frac{d \check{I}_{3}}{d t}=\left[\beta+\varepsilon \check{\beta}_{3}\right] S_{3} \check{\Theta}-\left[\mu+\varepsilon \mu_{3}\right] \check{I}_{3}-\left[\nu+\varepsilon \check{\nu}_{3}\right] \check{I}_{3} .
\end{aligned}
$$

The transformation of Andreasen and Christiansen (1993) is used to display the structure of system (3)-(5). For each disease class $Q$ we change the description from genotype numbers $\left(Q_{1}, Q_{2}, Q_{3}\right)$ into variables $\left(Q, p_{Q}, F_{Q}\right)$ that provides the total number of 
individuals in the class, $Q$, the frequency of the allele $a, p_{Q}$, and the deviation from Hardy-Weinberg proportions as measured by Wrights fixation index,

$$
F_{Q}=\frac{4 Q_{1} Q_{3}-Q_{2}^{2}}{\left(2 Q_{1}+Q_{2}\right)\left(2 Q_{3}+Q_{2}\right)}
$$

The total gene frequency is $p=\left(S p_{s}+\hat{I} \hat{p}_{I}+\check{I} \check{p}_{I}\right) / N$. The total number of infected individuals is $I=\hat{I}+\check{I}$ and we use the frequency $\pi=\hat{I} / I$ of disease type $v$ to describe the composition of the viral population. The structure of the terms of order $\varepsilon$ are not specified, but enter initially as functions $\psi_{Q}$ in the equation specifying $\dot{Q}$. The nine equations of system (3)-(5) then collect in four groups that describes the epidemic,

$$
\begin{aligned}
& \frac{d S}{d t}=b N-\mu S-\beta S I+\varepsilon \psi_{S}, \\
& \frac{d I}{d t}=\beta S I-(\nu+\mu)+\varepsilon \psi_{I},
\end{aligned}
$$

the composition of the pathogen population,

$$
\frac{d \pi}{d t}=\varepsilon \psi_{\pi}
$$

the gene frequencies in the three host classes,

$$
\begin{aligned}
\frac{d p_{S}}{d t} & =b \frac{\pi I}{S}\left(\hat{p}_{I}-p_{S}\right)+b \frac{(1-\pi) I}{S}\left(\check{p}_{I}-p_{S}\right)+\varepsilon \psi_{p S} \\
\frac{d \hat{p}_{I}}{d t} & =\beta S\left(p_{S}-\hat{p}_{I}\right)+\varepsilon \hat{\psi}_{p} \\
\frac{d \check{p}_{I}}{d t} & =\beta S\left(p_{S}-\check{p}_{I}\right)+\varepsilon \check{\psi}_{p}
\end{aligned}
$$

and the deviation from Hardy-Weinberg proportions in the three classes of the host population,

$$
\begin{aligned}
\frac{d F_{S}}{d t} & =b \frac{N}{S}\left(\frac{\left(p-p_{S}\right)^{2}}{p_{S} q_{S}}\left(1-F_{S}\right)-\frac{p q}{p_{S} q_{S}} F_{S}\right)+\varepsilon \psi_{F S}, \\
\frac{d \hat{F}_{I}}{d t} & =\beta S\left(\frac{\left(p_{S}-\hat{p}_{I}\right)^{2}}{\hat{p}_{I} \hat{q}_{I}}\left(1-\hat{F}_{I}\right)-\frac{p_{S} q_{S}}{\hat{p}_{I} \hat{q}_{I}}\left(\hat{F}_{I}-F_{S}\right)\right)+\varepsilon \hat{\psi}_{F}, \\
\frac{d \check{F}_{I}}{d t} & =\beta S\left(\frac{\left(p_{S}-\check{p}_{I}\right)^{2}}{\check{p}_{I} \check{q}_{I}}\left(1-\check{F}_{I}\right)-\frac{p_{S} q_{S}}{\check{p}_{I} \check{q}_{I}}\left(\check{F}_{I}-F_{S}\right)\right)+\varepsilon \check{\psi}_{F} .
\end{aligned}
$$

For no genetic variation in the parameters of the model, i. e. for $\varepsilon=0$, the host population settle at Hardy-Weinberg equilibrium and no change in the composition of the pathogen population is expected. The system (6)-(9) therefore contains a two dimensional, attracting mainfold of fixed points,

$\varpi(p, \pi)=\left\{\left(S, I, \pi, p_{S}, \hat{p}_{I}, \check{p}_{I}, F_{S}, \hat{F}_{I}, \check{F}_{I}\right)=\left(S^{*}, I^{*}, \pi, p, p, p, 0,0,0\right) \mid 0 \leq \pi \leq 1,0 \leq p \leq 1\right\}$, 
where $S^{*}$ and $I^{*}$ are the equilibrium values of $S$ and $I$ determined from (6) (see Appendix A). For small variation in the parameters of the model, $\varepsilon \ll 1$, the model has a twodimensional quasi steady state (Segel, 1988), and we can use the theorem of Thikonov (Hoppensteadt, 1966) to show that solutions to the model will move 'quickly' to be near the manifold $\varpi$. Once the solution is at a distance of order $\varepsilon$ to $\varpi$, all terms in equations (6)-(9) are of order $\varepsilon$, and the terms of order $O(\varepsilon)$ that describe the effect of genetic variation becomes important for the dynamics. The convergence to the manifold $\varpi$ is the 'fast' dynamics of the system, and close to $\varpi$ the rate of change is small, and we have reached the 'slow' dynamics of the system. The 'slow' dynamics is approximated by the dynamics on $\varpi$. The biological interpretation is that the epidemic and demographic processes will settle at an equilibrium virtually independent of the genetic composition of the population, that the frequency of $a$ will be virtually identical in all disease classes and that the genotypes will occur in frequencies indistinguishable from the Hardy-Weinberg proportions in all disease classes after a short initial transience. The remaining variables $\pi$ and $p$ describe the frequency of $v$ and $a$, respectively, and the slow dynamics on $\varpi$ therefore correspond to the slow coevolution of disease and host.

The change in the genetic composition of disease and host along the manifold $\varpi$ is well approximated by the solutions to

$$
\begin{gathered}
\dot{\pi}=\varepsilon \pi(1-\pi)\left(S\left\{\hat{\beta}_{i}-\check{\beta}_{i} \mid p\right\}+\beta S\left\{\hat{\tau}_{i}-\check{\tau}_{i} \mid p\right\}-\left\{\hat{\nu}_{i}-\check{\nu}_{i} \mid p\right\}\right) \\
\dot{p}=\varepsilon p q\left(-\left\langle\mu_{i} \mid p\right\rangle+\frac{(b-\beta S) I / S}{\beta S+b I / S}\left\langle\pi \hat{\beta}_{i} S+(1-\pi) \check{\beta}_{i} S \mid p\right\rangle\right. \\
\left.+\frac{b I / S}{\beta S+b I / S}\left\langle\pi \hat{\nu}_{i}+(1-\pi) \check{\nu}_{i} \mid p\right\rangle\right)
\end{gathered}
$$

on time intervals of the form $(0 ; T / \varepsilon)$ where $T$ may be replaced by $\infty$ if $(\pi, p)$ settles at a uniform asymptotically stable equilibrium. ${ }^{1}$ The variables $S, I$, and $N$ in these equations are evaluated at the epidemic equilibrium, and we have used the notation $\left\langle k_{i} \mid p\right\rangle=k_{1} p+k_{2}(q-p)-k_{3} q$ for the average excess of allele $a$ over allele $A$ in the genotypic values of parameter $k$ and the notation $\left\{k_{i} \mid p\right\}=k_{1} p^{2}+k_{2} 2 p q+k_{3} q^{2}$ for the population average of the genotypic values of parameter $k$ (Andreasen and Christiansen, 1993). Except for minor variations in the parametrization, this result was obtained by Beck (1984). However, the method in Appendix A is considerably easier than the method used by Beck.

\footnotetext{
${ }^{1}$ An equilibrium $y_{0}$ is uniform asymptotically stable if the deviations $y(t)-y_{0}$ can be uniformly bounded by a decaying exponential function over all initial conditions in the ball $\left|y-y_{0}\right|$.
} 


\section{The dynamics of weak selection}

Equation (10) resemble the classical equation for the weak selection approximation in diploid population genetics. To emphasize this we introduce the genotypic fitness coeffcients in a population infected only by the $v$ virus:

$$
\hat{s}_{i}=-\mu_{i}+\frac{(b-\beta S) I / S}{\beta S+b I / S} \hat{\beta}_{i}-\frac{b I / S}{\beta S+b I / S} \hat{\nu}_{i} \quad i=1,2,3,
$$

and we assume similar definitions, $\check{s}_{i}, i=1,2,3$, for the $V$ virus. These fitnesses depend on the epidemiology in a complicated way (Andreasen and Christiansen, 1993). The genotypic fitnesses in the classical sense are $\varepsilon\left(\pi \hat{s}_{i}+(1-\pi) \tilde{s}_{i}\right), i=1,2,3$, but we may drop the factor $\varepsilon$ and refer to the coefficients $\pi \hat{s}_{i}+(1-\pi) \check{s}_{i}, i=1,2,3$, as the genotypic fitnesses. The classical form of equation (10) is then

$$
\dot{p}=\varepsilon p q\left\langle\pi \hat{s}_{i}+(1-\pi) \tilde{s}_{i} \mid p\right\rangle .
$$

The corresponding homozygote fitness excess relative to the heterozygote are $\hat{r}_{1}=\hat{s}_{1}-\hat{s}_{2}$, $\hat{r}_{3}=\hat{s}_{3}-\hat{s}_{2}, \check{r}_{1}=\check{s}_{1}-\check{s}_{2}$ and $\check{r}_{3}=\check{s}_{3}-\check{s}_{2}$, and the average excess growth rate of allele $a$ is

$$
\hat{r}(p)=\left\langle\hat{s}_{i} \mid p\right\rangle=\hat{r}_{1} p-\hat{r}_{3} q \quad \text { and } \quad \check{r}(p)=\left\langle\check{s}_{i} \mid p\right\rangle=\check{r}_{1} p-\check{r}_{3} q
$$

in a host population exposed solely to virus type $v$ and type $V$, respectively. The dynamics of the gene frequency in the host therefore is determined by

$$
\dot{p}=\varepsilon p q(\pi \hat{r}(p)+(1-\pi) \check{r}(q)) .
$$

Equation (11) has the structure of the equation for the selection in haploid population genetics. The excess in fitness of virus $v$ over virus $V$ in a population comprised entirely of individuals of genotype $i$ is

$$
c_{i}=\left(\hat{\beta}_{i}-\check{\beta}_{i}\right) S+\left(\hat{\tau}_{i}-\check{\tau}_{i}\right) \beta S-\left(\hat{\nu}_{i}-\check{\nu}_{i}\right), \quad i=1,2,3,
$$

which is similar to the relative fitness excess above. These parameters bring equation (11) on the form

$$
\dot{\pi}=\varepsilon \pi(1-\pi)\left(c_{1} p^{2}+c_{2} 2 p q+c_{3} q^{2}\right) .
$$

Thus, at any time the change in frequency $\pi$ of the viral haploid $v$ is determined by the average excess in fitness of $v$ in the mixed host population, $c_{1} p^{2}+c_{2} 2 p q+c_{3} q^{2}$.

The transmission potential $R_{0}$ given by equation (1) is a measure of the competitive ability of a viral type, and in analogy with the competitive exclusion principle, one can show - under strong assumptions about the linearity of mortality and disease transmission - that the type with the highest value of $R_{0}$ will outcompete all other types. For a 
population comprised of only one genotype, the fitness parameters $c_{i}$ indicates the same since to first order we have

$$
\begin{aligned}
\hat{R}_{0}-\check{R}_{0} & =\varepsilon\left(\frac{(\hat{\beta}-\check{\beta}) N+(\hat{\tau}-\check{\tau}) \beta N}{\mu+\nu}-\frac{(\hat{\nu}-\check{\nu}) \beta N}{(\mu+\nu)^{2}}\right)+O\left(\varepsilon^{2}\right) \\
& =\varepsilon R_{0} \frac{1}{\mu+\nu}((\hat{\beta}-\check{\beta}) S+(\hat{\tau}-\check{\tau}) \beta S-(\hat{\nu}-\check{\nu}))+O\left(\varepsilon^{2}\right)
\end{aligned}
$$

where we used the equilibrium condition $\beta S=\mu+\nu$. Therefore, from the definition of $c_{i}$ we get that

$$
\frac{\hat{R}_{0}-\check{R}_{0}}{R_{0}}=\frac{\varepsilon c_{i}}{\mu+\nu}+O\left(\varepsilon^{2}\right),
$$

and we see that $c_{i}$ measures the relative difference in transmission potential for the two virus types per 'pathogen generation time' $(\mu+\nu)^{-1}$.

The detailed model and the weak selection model are compared on the basis of numerical solutions. The models are integrated using a fourth order Runge-Kutta algorithm with adaptive step-size. We assume that the slow variables $(\pi, p)$ do not change significantly during the initial transience in the detailed model, so the same initial values for $\pi$ and $p$ are used in both models as conventional in quasi-steady state approximations.

\subsection{Phase plane analysis}

The weak selection model given by equations (14) and (15) is a special case of the general coevolution models studied by, e. g., Levin and Udovic (1977). The present model is density independent and it has a specified structure in the type of frequency dependence, so more can be said about it than about general models. For instance, at a polymorhic coevolutionary equilibrium $\left(\pi^{*}, p^{*}\right)$ the heterozygote cannot have intermediate fitness (Levin and Udovic, 1977). In our case this follows immediately from the form of equation (12) where the genotypic fitnesses at equilibrium enter exactly as in the classical slow selection equation.

The $\dot{\pi}=0$ isoclines of the system may number 0,1 or 2 in addition to the two trivial null-isoclines $\pi=0$ and $\pi=1$ which always exists in a population genetic model without mutation. The non-trivial isoclines are from equation (15) of the form $p=p^{*}$, where $p^{*}$ is a root of the polynomial on the right side of equation (15) given by

$$
p^{*}=\frac{c_{3}}{-\left(c_{2}-c_{3}\right) \pm \sqrt{c_{2}^{2}-c_{1} c_{3}}}
$$

and subject to the constraint $0<p^{*}<1$. Similarly, the dynamics of $p$ has two absorbing states with only one allele present, namely $p=0$ and $p=1$. The remaining dynamics of $p$ is determined by the two linear functions $\hat{r}(p)$ and $\check{r}(p)$ in (13) that give the average excess fitnesses of $a$ on the two boundaries $\pi=0,1$. The sign of $\dot{p}$ is determined by a 
Table 1: The number of internal $\dot{\pi}=0$ isoclines for various values of $c_{i}$, the excess in fitness of virus $v$ over virus $V$ in a population comprised entirely of individuals of genotype i.

\begin{tabular}{|c|c|c|}
\hline & $c_{3}>0$ & $c_{3}<0$ \\
\hline$c_{1}>0$ & $\left.\begin{array}{ll}c_{2}>-\sqrt{c_{1} c_{3}}: & 0 \\
c_{2}<-\sqrt{c_{1} c_{3}}: & 2\end{array}\right\}$ & 1 \\
\hline$c_{1}<0$ & 1 & $\left.\begin{array}{l}c_{2}>\sqrt{c_{1} c_{3}}: \quad 0 \\
c_{2}<\sqrt{c_{1} c_{3}}: 2\end{array}\right\}$ \\
\hline
\end{tabular}

convex combination of $\hat{r}(p)$ and $\check{r}(p)$ in the interior. Thus for fixed $p$ at most one point given by

$$
\pi^{*}(p)=\frac{-\check{r}(p)}{\hat{r}(p)-\check{r}(p)}
$$

has $\dot{p}=0$, and this defines the $\dot{p}=0$ isocline. At most one point has $\dot{p}=0$ for fixed $\pi$, so the $\dot{p}=0$ isocline is also defined by

$$
p^{*}(\pi)=\frac{\hat{r}_{3} \pi+\check{r}_{3}(1-\pi)}{\left(\hat{r}_{1}+\hat{r}_{3}\right) \pi+\left(\check{r}_{1}+\check{r}_{3}\right)(1-\pi)}
$$

Therefore, the system can have 0,1 or 2 internal equilibria, at most one at each of the $\dot{\pi}=0$ isoclines, and an internal equilibrium with $p=p^{*}$ given by equation (16) has $\pi=\pi^{*}$ where $\pi^{*}=\pi^{*}\left(p^{*}\right)$ from equation (17). Because $0 \leq \pi^{*} \leq 1$ the equilibrium exists if and only if $\hat{r}\left(p^{*}\right) \check{r}\left(p^{*}\right) \leq 0$ from (17).

An internal equilibrium with gene frequency $p^{*}$ is present when the average excess in fitness of $a$ is of opposite signs in individuals infected by virus $v$ or by virus $V$ in a host population with gene frequency $p^{*}$. That is, the two virus types should induce the highest average fitness for different alleles. Equation (16) determines one valid frequency $p^{*}$ when $c_{1} c_{3} \leq 0$ and otherwise zero or two internal $\dot{\pi}=0$ isocline exists as shown in Table 1.

The stability of an internal equilibrium $\left(\pi^{*}, p^{*}\right)$ can be investigated by a standard linear analysis giving the following linearization

$$
\left(\begin{array}{c}
\dot{p} \\
\dot{\pi}
\end{array}\right)=D\left(\begin{array}{c}
p \\
\pi
\end{array}\right)
$$

where

$$
\boldsymbol{D}=\left(\begin{array}{cc}
0 & \pm 2 \pi^{*}\left(1-\pi^{*}\right) \sqrt{c_{2}^{2}-c_{1} c_{3}} \\
p^{*}\left(1-p^{*}\right)\left(\hat{r}\left(p^{*}\right)-\check{r}\left(p^{*}\right)\right) & p^{*}\left(1-p^{*}\right)\left(\left(\hat{r}_{1}+\hat{r}_{3}\right) \pi^{*}+\left(\check{r}_{1}+\check{r}_{3}\right)\left(1-\pi^{*}\right)\right)
\end{array}\right)
$$




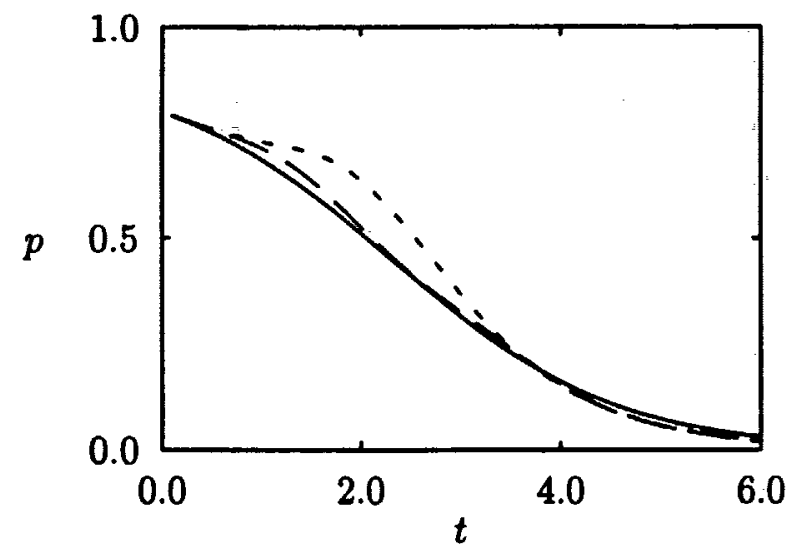

Figure 1: The solution of the approximate model (solid curve) and of the full model with $\varepsilon=0.2$ (broken curve) and 0.5 (dotted curve) in a situation where $a$ fixes in the host population and $V$ fixes in the virus population. The parameter values are $b=0.2, \mu=0.1$ and $\nu=\beta=1$. The genetically determined parameter perturbations are $\mu_{1}=-\mu_{3}=1.0, \check{\beta}_{1}=-\hat{\beta}_{3}=-1.0$, and $\check{\nu}_{1}=-\hat{\nu}_{3}=-2.0$ while all other perturbations are 0 .

\subsection{Analysis of equilibria}

When the polynomial $c_{1} p^{2}+c_{2} 2 p q+c_{3} q^{2}$ has no roots between 0 and 1 one strain of virus has the highest growth rate for all host populations. Therefore, $\pi$ is monotonically increasing or decreasing in the open unit square and one of the lines $\pi=0$ and $\pi=1$ will be attracting. On this line the dynamics is determined by selection in the host population exposed to just one viral type, the situation discussed by Andreasen and Christiansen (1993). The equilibrium attracts all solutions starting in the interior of the unit square at an exponential rate and the full system will remain $O(\varepsilon)$-close to the approximate solution for all time. Figure 1 shows an example where the only stable equilibrium is fixation on genotype $a a$ and virus type $V$. The approximation seems to work quite well for perturbations as large as $\varepsilon=50 \%$.

The case $c_{1} c_{3}<0$, with one internal $\dot{\pi}=0$ isocline at $p=p^{*}$, includes most of the interesting coevolutionary behavior of the system, and we discuss this case in some detail. When $c_{1} c_{3}<0$ one virus type will have the highest growth rate in a monomorphic population consisting of $a a$ and the other viral strain will be most fit in a population consisting entirely of $A A$. We may assume that $v$ has an advantage on aa, and that $V$ grows the fastest on $A A$, i. e., $c_{1}>0$ and $c_{3}<0$. This means that $\dot{\pi}>0$ for $p>p^{*}$ and $\dot{\pi}<0$ for $p<p^{*}$, and that the appropriate solution in (16) uses the minus sign in the denominator.

The existence of the internal equilibrium and its local stability depend on $\hat{r}\left(p^{*}\right)$ and $\check{r}\left(p^{*}\right)$ as indicated in Table 2. If $\hat{r}\left(p^{*}\right) \check{r}\left(p^{*}\right)>0$ then $p$ is monotone and the system will possess one stable equilibrium with monomorphism in both host and pathogen. We 
Table 2: Existence and stability conditions for the internal equilibrium in model (14)-(15) when $c_{1}>0$ and $c_{3}<0$, i. e., when one internal $\dot{\pi}=0$ isocline exists (Table 1 ).

\begin{tabular}{lcc}
\hline & $\hat{r}\left(p^{*}\right)>0$ & $\hat{r}\left(p^{*}\right)<0$ \\
\hline$\tilde{r}\left(p^{*}\right)>0$ & no equilibrium & focus \\
$\tilde{r}\left(p^{*}\right)<0$ & saddle & no equilibrium \\
\hline
\end{tabular}

will not discuss this situation further and focus on the cases where $\hat{r}\left(p^{*}\right)$ and $\check{r}\left(p^{*}\right)$ have opposite sign. We distinguish between two situations. First, the situation where $\hat{r}\left(p^{*}\right)>0$ and $\check{r}\left(p^{*}\right)<0$. Then at equilibrium allele $a$ has the higher average fitness on $v$ and $A$ the higher on $V$, and the combinations $a$ with $v$ and $A$ with $V$ provide the higher fitness for both host and pathogen. The internal equilibrium is a saddle according to Table 2 . Second, we consider the situation where $\hat{r}\left(p^{*}\right)<0$ and $\check{r}\left(p^{*}\right)>0$. Then at equilibrium allele $a$ has the higher average fitness on $V$ and $A$ the higher on $v$, and the higher fitness for host and pathogen is obtained in different combinations. The internal equilibrium produced is a focus.

\subsubsection{Polymorphic equilibria of the saddle type}

The internal equilibrium is unstable in this situation, so monomorphism in the pathogen always result. We first consider the case where $a$ has the higher average fitness for high frequencies of $v$ and that $V$ and $A$ also induce high fitnesses for each other, i. e., $\hat{r}(p)>0$ and $\check{r}(p)<0$ for all $p, 0<p<1$, and not only for $p=p^{*}$. In the phase portrait (Fig. 2) the $\dot{p}=0$ isocline passes from the line $p=0$ to the line $p=1$ (the solid curve in Fig. 2). The equilibria at $(0,0)$ and $(1,1)$ are asymptotically stable while the equilibria at $(1,0)$ and $(0,1)$ are unstable, and the stable manifolds $\mathcal{S}$ of the internal equilibrium separate the basins of attraction for the two stable equilibria with $(1,0)$ and $(0,1) \in \mathcal{S}$. The system will go to monomorphism in both pathogen and host, $a$ with $v$ or $A$ with $V$, with the outcome depending on initial conditions.

Disease induced polymorphism in the host may occur if $\hat{r}_{1}=\hat{r}(1)<0$, but $\hat{r}\left(p^{*}\right)>0$, because this implies that $\hat{r}_{3}<0$. Therefore, the host show overdominant selection with only virus $v$, and the equilibrium with frequency $p^{\dagger}=\hat{r}_{3} /\left(\hat{r}_{1}+\hat{r}_{3}\right)$ is stable as long as virus $V$ does not occur in the population. The $\dot{p}=0$ isocline bends to the right and crosses the line $\pi=1$ at $p=p^{\dagger}$ (broken curve in Fig. 2). When $\hat{r}_{1}=0$ the $\dot{p}=0$ isocline passes through the equilibrium at $(1,1)$ which bifurcates and exchanges stability with the equilibrium $\left(1, p^{\dagger}\right)$ as $\hat{r}_{1}$ becomes negative. For $p^{\dagger}>p^{*}$ the equilibrium $\left(1, p^{\dagger}\right)$ remains 


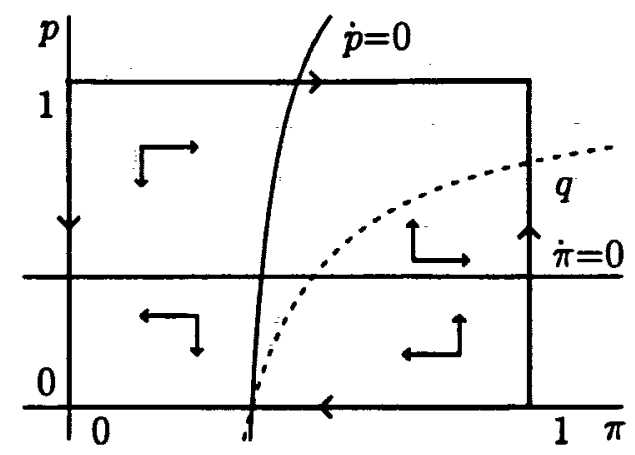

Figure 2: Phase portrait for model (14)-(15) with $\hat{r}\left(p^{*}\right)>0$ and $\check{r}\left(p^{*}\right)<0$, where the internal equilibrium is a saddle. The solid curve shows a situation where monomorphism prevails in a host population that is infected only with one virus strain. The broken curve shows a situation where polymorphism in the host is possible in a population only infected with virus $v$.

stable, and at $p^{\dagger}=p^{*}$ the equilibrium fuses with the internal equilibrium and looses its stability. For $p^{\dagger}<p^{*}$ the internal equilibrium does not exist, $\left(1, p^{\dagger}\right)$ is unstable to the introduction of $V$ and $(0,0)$ is the only stable equilibrium. Similarly the equilibrium at $(0,0)$ may exchange stability with an equilibrium corresponding to host polymorphism in the state where only $V$ is present. The two equilibria with host polymorphism and pathogen monomorphism may exist and be stable simultaneously.

The comparison between solutions to the full model and the approximate solutions is somewhat delicate when the internal equilibrium is a saddle (Fig. 3). For solutions starting far from $\mathcal{S}$, one of the fixation states is uniform asymptotically stable, and the two solutions remain close in both a quantitative and a qualitative sense. For solutions starting close to the stable manifold the approximate and the full solutions may differ in two ways. They may simply fall in different basins of attraction because of a small difference in the position of the stable manifolds in the full model and in the approximate model. Even when the curves remain in the same basin of attraction, one trajectory may be slower than the other. The trajectories are stalled in the neighborhood of the saddlepoint, so curves that come close to the saddle slow down compared to curves that stay farther away, therefore near $\mathcal{S}$ we obtain only qualitative correspondence between the solutions. With these reservations we conclude that the approximate model is a good predictor of the coevolutionary behavior in the full model.

\subsubsection{Polymorphic equilibria of the focus type}

Again we first assume that the average excess fitnesses of allele $a$ have the same sign for all gene frequencies, i. e., we assume that $\hat{r}(p)<0$ and $\check{r}(p)>0$ for all $p, 0<p<1$. Thus, $a$ has the higher average fitness for high frequencies of $V$ and $A$ is favoured for high 


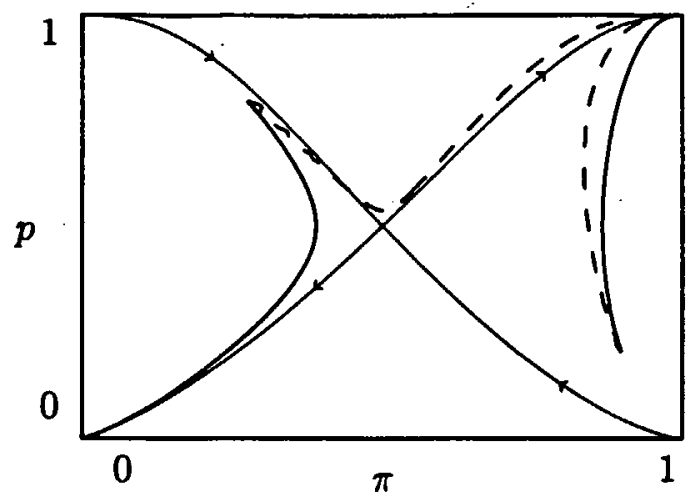

Figure 3: Behavior of the coevolution model in a situation where the fixed point for coexistence is a saddle. Trajectories for the approximate model (solid lines) and the full model (broken lines) may separate if initial conditions are near the stable manifold of the fixed point for the approximate model (thin lines). The parameter values are the same as in Figure 1. The genetically determined variation in parameters for $a a$ is $\mu_{1}=0.00125, \check{\nu}_{1}=-\hat{\nu}_{1}=0.25$, and $\check{\beta}_{1}=-\hat{\beta}_{1}=0.22$. For $A A$ the deviations of $\nu$ and $\beta$ are $\check{\nu}_{3}=-\hat{\nu}_{3}=-0.13$, and $\check{\beta}_{3}=-\hat{\beta}_{3}=$ -0.11 while $\mu_{3}=\mu_{1}$. Deviations for the heterozygote are set to zero. The values correspond to $\operatorname{tr} D=-0.0625$ and $\operatorname{det} D=-0.5$.

frequencies of $v$. The phase portrait of this situation is shown in Fig. 4, and the $\dot{p}=0$ isocline passes from the line $p=0$ to the line $p=1$ (the solid curve in Fig. 4). All four corners are saddles, and the edges connect these four saddles in a heteroclinic orbit, $\gamma$, strung between the saddle points in $\Gamma=\{(0,0),(0,1),(1,1),(1,0)\}$. The solutions will rotate clockwise around the internal equilibrium.

The stability of the internal equilibrium is determined by the trace condition $\operatorname{tr} D<0$ which is

$$
\left(\hat{r}_{1}+\hat{r}_{3}\right) \pi^{*}+\left(\check{r}_{1}+\check{r}_{3}\right)\left(1-\pi^{*}\right)<0 .
$$

Thus, the polymorhic equilibrium is stable when the denominator in the equilibrium gene frequency in the host, equation (18), is negative. This happens when fitnesses $\pi^{*} \hat{s}_{i}+\left(1-\pi^{*}\right) \check{s}_{i}, i=1,2,3$, are overdominant at equilibrium, i. e. when the fitness of the heterozygote at equilibrium is larger than the fitness of either homozygote (Levin and Udovic, 1977). Equation (18) simplifies the stability conditions (19) to

$$
\check{r}_{1} \hat{r}_{3}<\hat{r}_{1} \check{r}_{3}
$$

When condition (20) is satisfied damped oscillations occur, and a Hopf-type bifurcation is expected to take place at $\operatorname{tr} \boldsymbol{D}=0$ when the polymorhic equilibrium becomes unstable. However, the system given by equations (14) and (15) is degenerate, in that the third order terms that determine the nature of the Hopf-bifurcation vanish. 


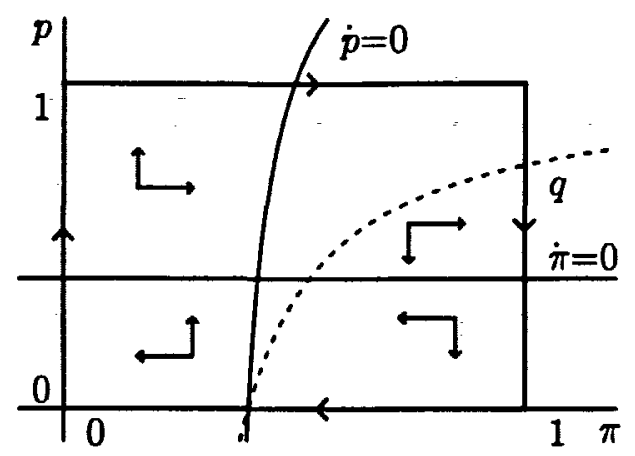

Figure 4: Phase portrait for model (14)-(15) with $\hat{r}\left(p^{*}\right)<0$ and $\check{r}\left(p^{*}\right)>0$, where the internal equilibrium is a focus. The solid curve shows a situation where monomorphism prevails in a host population that is infected only with one virus strain. In this situation the edges form a heteroclinic orbit. The broken curve shows a situation where polymorphism in the host is possible in a population only infected with virus $v$.

The system undergoes a global bifurcation at the heteroclinic orbit $\gamma$ simultaneously with the Hopf bifurcation. Solutions near $\gamma$ will be attracted to $\gamma$ when $B<1$, where

$$
B=\prod_{q \in \Gamma} \frac{\lambda_{u}(q)}{-\lambda_{s}(q)}
$$

and $\lambda_{s}(q)$ and $\lambda_{u}(q)$ are the stable and the unstable eigenvalues of the linearization around the saddle point $q \in \Gamma$ (Guckenheimer and Holmes, 1983). Evaluating these eigenvalues we get

$$
B=\frac{\check{r}_{3}}{c_{3}} \frac{c_{3}}{\check{r}_{1}} \frac{\hat{r}_{1}}{c_{1}} \frac{c_{1}}{\hat{r}_{3}}=\frac{\check{r}_{3} \hat{r}_{1}}{\check{r}_{1} \hat{r}_{3}}
$$

Thus, $B<1$ if and only if condition (20) is not satisfied, and so the boundary orbit $\gamma$ is attracting exactly when the internal equilibrium $\left(\pi^{*}, p^{*}\right)$ is repelling. The internal equilibrium and the heteroclinic orbit are therefore neutrally stable at $\operatorname{tr} D=0$, and in passing through tr $\boldsymbol{D}=0$ the system goes directly from solutions spiraling slowly into $\left(\pi^{*}, p^{*}\right)$ to solutions spiraling slowly away from the equilibrium and onto the heteroclinic orbit.

When $\operatorname{tr} \boldsymbol{D}<-O(\varepsilon)$, the polymorphic equilibrium is uniform-asymptotically stable, damped oscillations occur, and the solutions for the full and approximate models stay close for all time (Fig. 5). The system does not have a uniform-asymptotically stable solution, however, in the case of undamped oscillations when $\operatorname{tr} D>O(\varepsilon)$. The full and approximate solutions will diverge over time, but the divergence is essentially due to differences in the period of the undamped oscillation. The solutions oscillate out onto the heteroclinic orbit, and we maintain a qualitative correspondence between the approximate and the full solutions. 


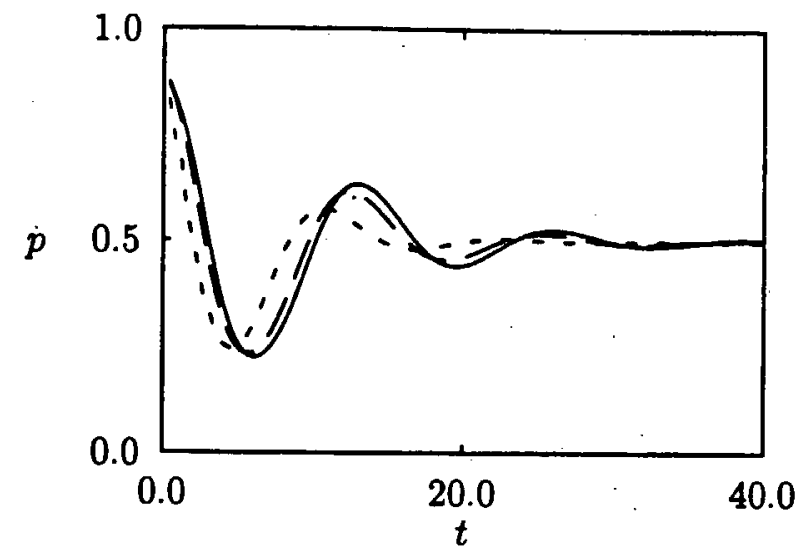

Figure 5: Solutions to the approximate model (broken curve $\varepsilon=0.1$, dotted curve $\varepsilon=0.2$ ) and the full model (solid curve) in the situation where a stable double polymorphic coevolutionary equilibrium exists. The parameter values are the same as in Figure 1. The genetically determined parameter perturbations are $\mu_{1}=\mu_{3}=0.5, \hat{\beta}_{1}=-\check{\beta}_{1}=-\hat{\beta}_{3}=\check{\beta}_{3}=8.4$, and $\hat{\nu}_{1}=-\check{\nu}_{1}=$ $-\hat{\nu}_{3}=\check{\nu}_{3}=8.8$ with all other perturbation equal 0 . The values correspond to tr $D=-0.25$ and $\operatorname{det} D=0.25$.

When tr $D=O(\varepsilon)$ neither the quantitative nor the qualitative behavior of the full system is determined by the approximate model. The approximate model change from damped to undamped oscillations through a critical Hopf bifurcation, but the full system undergoes a usual (sub- or supercritical) Hopf bifurcation giving rise to a limit cycle which disappears through a global bifurcation at the heteroclinic orbit (Fig. 6).

As before the $\dot{p}=0$ isocline may bend, as indicated by the broken curve in Fig. 4 . This gives rise to an equilibrium with polymorphism in the host for one or both of the monomorphic virus populations, $\left(0, p^{\ddagger}\right)$ and $\left(1, p^{\dagger}\right)$. If $1>p^{\dagger}>p^{*}$ then the equilibrium $(1,1)$ is locally stable, and when the internal equilibrium exist $\left(0 \leq p^{\ddagger}<p^{*}\right)$, it is unstable. Thus, the system cannot have two stable equilibria when the unique internal equilibrium is a focus.

\subsubsection{Two polymorphic equilibria}

When two $\dot{\pi}=0$ isoclines exist the phase portrait essentially consists of combinations of the situations described above. When two internal equilibria exists, one is a saddle and one is a focus. A heteroclinic orbit cannot exist, and a stable boundary equilibrium always exists. The essentially new phenomenon that may occur is simultaneous stability of the focus and a boundary equilibrium with one virus type and either monomorphism or polymorphism in the host. 

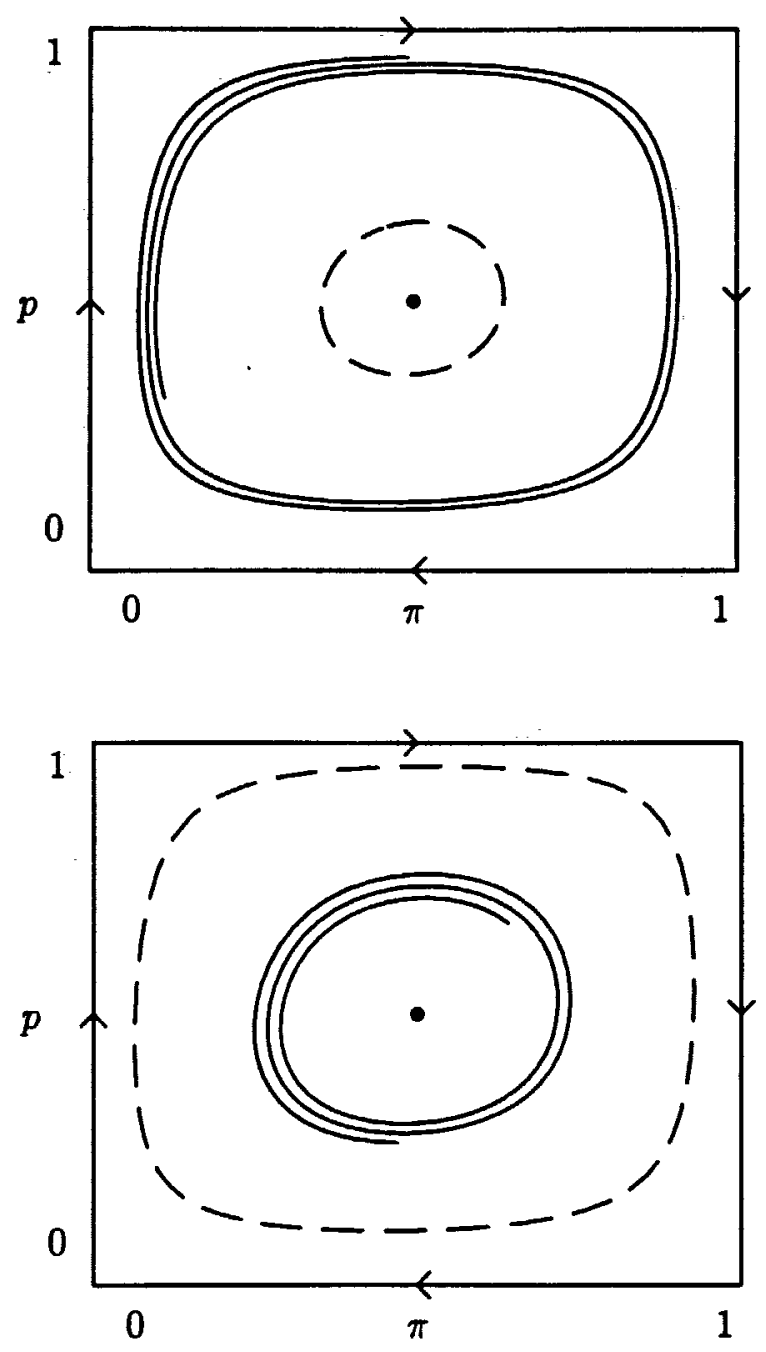

Figure 6: The dynamics of the of the full model is sensitive to small changes in $\varepsilon$ when $\operatorname{tr} D=$ $O(\varepsilon)$. The fixed point at $\left(\frac{1}{2}, \frac{1}{2}\right)$ undergoes a subcritical Hopf bifurcation at $\varepsilon \approx 0.0092$ spawning an unstable limit cycle that disappears in a global bifurcation at the heteroclinic orbit for $\varepsilon \approx 0.05$. - Top diagram: $\varepsilon=0.0095$; bottom diagram: $\varepsilon=0.014$. - The parameter values are the same as in Figure 1. The genetically determined variation in parameters for $a a$ is $\mu_{1}=0.00075, \check{\nu}_{1}=-\hat{\nu}_{1}=-0.13$, and $\check{\beta}_{1}=-\hat{\beta}_{1}=0.12$. For $A A$ the deviations of $\nu$ and $\beta$ are $\check{\nu}_{3}=-\hat{\nu}_{3}=0.05$, and $\bar{\beta}_{3}=-\hat{\beta}_{3}=0.05$ while $\mu_{3}=\mu_{1}$. Deviations for the heterozygote are set to zero. The values correspond to $\operatorname{tr} D=0.0375$ and $\operatorname{det} D=0.25$. 


\subsection{Degeneracy}

The local stability of the double polymorphic equilibrium $\left(\pi^{*}, p^{*}\right)$ need to be studied in more detail to understand the biological origin of the degeneracy in the model. The model given by equations (14) and (15) has the form

$$
\begin{aligned}
& \dot{\pi}=\pi(1-\pi) f(p ; \vartheta), \\
& \dot{p}=p(1-p) g(\pi, p ; \vartheta),
\end{aligned}
$$

where $\vartheta$ is a bifurcation parameter. The function $f(p ; \vartheta)$ is independent of $\pi$, i. e., $f_{\pi}^{\prime} \equiv 0$, and the function $g(\pi, p ; \vartheta)$ is linear in $p$ and $\pi$, i. e., $g_{\pi \pi}^{\prime \prime} \equiv 0$ and $g_{p p}^{\prime \prime} \equiv 0$. For simplicity we focus on a single bifurcation parameter $\vartheta$ : At the bifurcation value $\vartheta=\vartheta_{0}$ we find that a pair of complex eigenvalues $\lambda(\vartheta)$ of the Jacobian

$$
\boldsymbol{D}(\vartheta)=\left(\begin{array}{cc}
0 & \pi^{*}\left(1-\pi^{*}\right) f_{p}^{\prime}\left(p^{*} ; \vartheta_{0}\right) \\
p^{*}\left(1-p^{*}\right) g_{\pi}^{\prime}\left(\pi^{*}, p^{*} ; \vartheta_{0}\right) & \pi^{*}\left(1-\pi^{*}\right) g_{p}^{\prime}\left(\pi^{*}, p^{*} ; \vartheta_{0}\right)
\end{array}\right)
$$

passes through the imaginary axis. In other words we have that

$$
\lambda\left(\vartheta_{0}\right)= \pm \mathrm{i} \omega \quad \text { and }\left.\quad \operatorname{Re} \frac{d \lambda}{d a}\right|_{\vartheta_{0}} \neq 0 .
$$

Thus, the second diagonal term of $\boldsymbol{D}$ vanishes at the bifurcation point, and we have that $g_{p}^{\prime}=0$.

It is well known that under conditions (22) a Hopf bifurcation occurs at $\left(\pi^{*}, p^{*} ; \vartheta_{0}\right)$, and this may be of three types:

Supercritical Hopf bifurcation: a stable periodic orbit occurs when the eigenvalues have positive real part and no periodic orbit exists when the eigenvalues have negative real part.

Subcritical Hopf bifurcation: an unstable periodic orbit occurs when the eigenvalues have negative real part and no periodic orbit exists when the eigenvalues have positive real part.

Critical Hopf bifurcation: the effect of the third order terms vanishes and the dynamics is determined by higher order terms.

The details of the bifurcation are determined by the third order terms which can be found by computing the normal form of the equation (Guckenheimer and Holmes, 1983). However, Liu et al. (1986) provide a formula that allows us to compute directly a critical parameter $C$, the sign of which in combination with the derivative $\operatorname{Re} \lambda^{\prime}\left(\vartheta_{0}\right)$ determines the type of the bifurcation. The assumptions about $f$ and $g ; f_{\pi}^{\prime} \equiv g_{\pi \pi}^{\prime \prime} \equiv g_{p p}^{\prime \prime} \equiv 0$ in particular, make $C$ vanish and the bifurcation is critical. 
This degeneracy implies that the model given by equations (14) and (15) is structurally unstable, i. e. even small changes in the vector field may give rise to significant changes in the phase portrait (Guckenheimer and Holmes, 1983). The model is based on a series of simplifying assumptions, and we will discuss how the model may be modified in order to remove the degeneracy.

The model is obtained as a first order approximation to a larger system of equations, so higher order terms may remove the degeneracy. Numerical solution of the full model (3)(5) show that the Hopf bifurcation and the global bifurcation at $\gamma$ are indeed separated in parameter space. A sub- or supercritical Hopf bifurcation occurs at the polymorphic equilibrium and gives rise to a limit cycle that undergoes a global bifurcation at the heteroclinic orbit, but both events happen for parameter values with $\operatorname{tr} D=O(\varepsilon)$. The transition between the two dynamical states of the model therefore occurs within a tiny area of parameter space. Thus, the model is structurally unstable in the sense that small changes in the vector field may alter the dynamics significantly if the changes outweighs the higher order terms in $\varepsilon$.

This return the focus to the approximate model given by equations (14) and (15). From our analysis the degeneracy depends on three properties of the model:

1. $f_{\pi}^{\prime}=0$, no frequency dependence in the fitness of the haploid pathogen.

2. $g_{p p}^{\prime \prime}=0$, no frequency dependence in the genotypic fitnesses of the diploid host (the allelic fitnesses are linear in host gene frequency).

3. $g_{\pi \pi}^{\prime \prime}=0$, the genotypic fitness of the diploid host is linear in the type frequencies of the virus population.

The selection on the variation in the host and pathogen populations is frequency dependent in the full model (3)-(5). The frequency dependence, however, is weak in the sense that, e. g., the frequency dependent effect in the host is of order $\varepsilon^{2}$ which is an order of magnitude lower than the effect described in the fitnesses $\varepsilon s_{i}$ of the approximate model.

The first property appears to be quite fundamental to SIR-type interactions which implies that the model allows for no direct frequency dependent selection among viral types. The expression for $f$ comes from the equation for the change in $\pi$ which from equation (28) of Appendix $A$ is of the form

$$
\frac{d \pi}{d t}=\varepsilon \pi(1-\pi)\left(\frac{\hat{\psi}_{I}}{\hat{I}}-\frac{\check{\psi}_{I}}{\check{I}}\right),
$$

where $\hat{\psi}_{I}$ and $\check{\psi}_{I}$ denotes the order $\varepsilon$-terms of $d \hat{I} / d t$ and $d \check{I} / d t$ evaluated at the slow manifold $\varpi$. The term $\hat{\psi}_{I} / \hat{I}$ is independent of $\pi$ since $\hat{\theta}$ in the infection rate and $\hat{I}$ in the removal rate are proportional to $\hat{I}=\pi I$. Thus, latent period, immunity, or densitydependence do not affect the degeneracy provided a quasi-equilibrium exists where the distribution of infections follows the frequencies of $v$ and $V$ in the population. 
If disease transmission is determined by heterogeneous mixing among hosts, like the mixing characteristic of sexually transmitted diseases in man, the infection rate is more complicated. However, frequency dependent selection among viral types still does not occur if exchange among groups is sufficiently strong and if reproduction is by random mating in the total population. To illustrate this assume that the host population is divided into two subpopulations $X$ and $Y$ with contact rates $c_{X}$ and $c_{Y}$. If the mixing is proportionate (Babour, 1978) the rate of new $v$-infections for genotype $i$ in subpopulation $X$ becomes

$$
\hat{\mathcal{L}}_{X i}=\left(\beta+\varepsilon \hat{\beta}_{i}\right) c_{X} S_{A i} \frac{c_{X} \hat{I}_{X}+c_{Y} \hat{I}_{Y}}{c_{X} N_{X}+c_{Y} N_{Y}}
$$

(Hethcote and Yorke, 1981). Births are divided among the two subpopulations in fixed proportions and otherwise the model is the SI-model of Section 2. The coordinate transformation in Section 2 for each subpopulation yield the dynamics of the type frequencies $\pi_{X}$ and $\pi_{Y}$ in subpopulation $X$ and $Y$ :

$$
\begin{aligned}
\dot{\pi}_{X} & =\beta S_{X} \frac{c_{X} c_{Y}}{c_{X} N_{X}+c_{Y} N_{Y}} \frac{I_{Y}}{I_{X}}\left(\pi_{Y}-\pi_{X}\right)+\varepsilon \psi_{\pi X}, \\
\dot{\pi}_{Y} & =\beta S_{Y} \frac{c_{X} c_{Y}}{c_{X} N_{X}+c_{Y} N_{Y}} \frac{I_{X}}{I_{Y}}\left(\pi_{X}-\pi_{Y}\right)+\varepsilon \psi_{\pi Y} .
\end{aligned}
$$

If the model has a stable endemic equilibrium then $\left(\pi_{X}, \pi_{Y}\right)$ settle to the slow manifold $(\pi, \pi)$. At this quasi-equilibrium the infection rate $\hat{\mathcal{L}}_{X i}$ again is proportional to $\pi$ and frequency dependent selection among viral types is excluded. More complicated mixing patterns such as preferred mixing (Jacquez et al., 1988; Blythe and Castillo-Chavez, 1989) do not affect this conclusion.

\section{Frequency dependent selection in the virus}

Direct interaction among viral types may cause frequency dependent selection in the viral population. To illustrate this we analyze a modified version of a model of phage-bacterium coevolution (Levin et al., 1977; Levin and Lenski, 1983; Stuart and Levin, 1984; Levin, 1988). In the phage-bacterium system a free living stage of the phages attack uninfected as well as infected bacteria, and in Levin's model genetic variation in attack rates allow for structural stability in the coevolutionary model.

A similar situation occurs for baculo vira infecting insect larvae. Baculo vira replicate rapidly and fill the entire body of the larvae with virus capsules with a protective proteinaceous cover. This shield allows the virus to stay active in the environment for as long as ten years. Infections primarily occur when larvae feed on leaves contaminated with virus capsules (Fraenkel-Conrat, Kimball and Levy, 1988). 
The pool of free virus capsules $W$ plays a central role in the transmission dynamics of baculo virus, and Anderson and May (1981) included it in the SI-model:

$$
\begin{aligned}
\dot{S} & =b N-\mu S-\beta S W \\
\dot{I} & =\beta S W-(\mu+\nu) I \\
\dot{W} & =\lambda I-\beta N W-\varrho W
\end{aligned}
$$

where $N=S+I$ is the total number of larvae. The amount of free virus produced per infected host that survive the infected stage is $\lambda / \nu$. Larvae meet virus capsules at the rate $\beta W$, and the amount of virus in the environment is measured in units of the amount ingested by a susceptible larvae to become infected. The term $\beta N W$ reflects the fact that both healthy and infected larvae eat and remove virus particles from the pool of free virus. This phenomenon induces a direct interaction between infection types because virus eaten by an already infected larvae is less likely to reproduce.

The disease persist in the population only if the number of infective doses produced by an infected larva, $\lambda /(\nu+\mu)$, times the probability that the virus is consumed by larvae $\beta N /(\beta N+\varrho)$ exceeds unity in a population where all larvae are susceptible, i. e.

$$
R_{0}=\frac{\lambda}{\mu+\nu} \frac{\beta N}{\beta N+\varrho}>1 .
$$

With this threshold condition satisfied an endemic equilibrium exists, and it may be stable or spawn a stable limit cycle through a Hopf bifurcation. To simplify the discussion we assume that the model has a stable endemic equilibrium. The non-stationary case can be handled in a similar way by using suitable time averages (Andreasen and Christiansen, 1993).

The full coevolutionary model with two viral types and two alleles to determine host response is given in Appendix B. After transformations, like those used in Section 2, the slow coevolution of host and pathogen is described by

$$
\begin{aligned}
\dot{\pi}=\varepsilon \pi(1-\pi)( & -\frac{\lambda S I-\beta W S^{2}}{I}\left\{\hat{\beta}_{i}-\check{\beta}_{i} \mid p\right\}-\frac{\lambda I}{W}\left\{\hat{\nu}_{i}-\check{\nu}_{i} \mid p\right\} \\
& +\frac{\beta S}{\lambda I / W+\beta S W / I}\left\{\hat{\lambda}_{i}-\check{\lambda}_{i} \mid p\right\}-(\hat{\varrho}-\check{\varrho}) \\
& \left.+\frac{\beta S W}{\lambda I / W+\beta S W / I}\left(\pi\left\{\hat{\beta}_{v i}-\check{\beta}_{v i} \mid p\right\}+(1-\pi)\left\{\hat{\beta}_{V i}-\check{\beta}_{V i} \mid p\right\}\right)\right) \\
\dot{p}=\varepsilon p(1-p)( & -\left\langle\mu_{i} \mid p\right\rangle+\frac{(b-\beta S W / I) W / S}{\beta S W / I+b I / S}\left(\pi\left\langle\hat{\beta}_{i} S \mid p\right\rangle+(1-\pi)\left\langle\check{\beta}_{i} S \mid p\right\rangle\right) \\
& \left.+\frac{b I / S}{\beta S W / I+b I / S}\left(\pi\left\langle\hat{\nu}_{i} \mid p\right\rangle+(1-\pi)\left\langle\check{\nu}_{i} \mid p\right\rangle\right)\right) .
\end{aligned}
$$


The transmission rate $\beta$ vary with genotype and viral type in both susceptible and infected larvae. The deviation from $\beta$ for susceptible larvae of genotype $i$ as before is described by $\hat{\beta}_{i}$ and $\dot{\beta}_{i}$ for infections of $v$ and $V$. The deviation from $\beta$ for larvae of genotype $i$ infected with virus type $v$ is described by $\hat{\beta}_{v i}$ and $\check{\beta}_{v i}$ when the lavae meet virus capsules of type $v$ and type $V$, respectively. The rate deviation for larvae of genotype $i$ infected with virus type $V$ is described by $\hat{\beta}_{V i}$ and $\check{\beta}_{V i}$.

Compared to model (14)-(15) the fitness of the virus now includes a frequency dependent term proportional to $\pi\left\{\hat{\beta}_{v i}-\check{\beta}_{v i} \mid p\right\}+(1-\pi)\left\{\hat{\beta}_{V i}-\check{\beta}_{V i} \mid p\right\}$. Thus, frequency dependent selection occurs in the viral population if $\hat{\beta}_{V i}-\check{\beta}_{V i} \neq \hat{\beta}_{v i}-\check{\beta}_{v i}$, i. e., if the two viral types induces different search behaviour in the infected hosts and if they differ in the amount or distribution of virus capsules produced. For example, if the feeding rate of lavae infected with strain $v$ is reduced and in addition the spatial distribution of virus is heterogeneous in that lavae infected with $v$ are more likely to encounter $v$-virus particles than $V$-particles, then genuine frequency dependence occurs and the degeneracy is broken.

The structural similarity between model (24)-(25) and model (14)-(15) is exposed by the introduction of aggregate parameters:

$$
\begin{aligned}
& \dot{\pi}=\varepsilon \pi(1-\pi)\left(c_{1} p^{2}+c_{2} 2 p q+c_{3} q^{2}-(\hat{\varrho}-\check{\varrho})+\pi\left(d_{1} p^{2}+d_{2} 2 p q+d_{3} q^{2}\right)\right) \\
& \dot{p}=\varepsilon p q\left(\pi\left(\hat{r}_{1} p-\hat{r}_{3} q\right)+(1-\pi)\left(\check{r}_{1} p-\check{r}_{3} q\right)\right) .
\end{aligned}
$$

We need not analyze the system in general to argue that the degeneracy is removed. Rather, we consider a highly symmetric situation, where the variation in encounter rates for infected individuals is independent of genotype, $d_{i}=d$, and where the fitness of hosts and virus are symmetric in that $c_{1}=-c_{3}=c, c_{2}=0, \hat{r}_{1}=\check{r}_{3}=-\alpha, \hat{r}_{3}=\check{r}_{1}=1$ and $\hat{\varrho}-\check{\varrho}=0$, where $c \geq 0$ and $\alpha \geq 0$ :

$$
\begin{aligned}
& \dot{\pi}=\varepsilon \pi(1-\pi)(c(p-q)+d \pi) \\
& \dot{p}=\varepsilon p q(-\pi(\alpha p+q)+(1-\pi)(p+\alpha q)) .
\end{aligned}
$$

For $d=0$ this corresponds to $c_{1} c_{3} \leq 0, \hat{r}(p)=-(\alpha p+q) \leq 0$ and $\check{r}(p)=p+\alpha q \geq 0$, which is the focus case depicted in Fig. 4. We restrict attention to the case where $-\frac{1}{2} c<d<\frac{1}{2} c$. This ensures that Fig. 4 still represents the phase portrait with the existence of exactly one internal equilibrium of focus type and a heteroclinic orbit with clockwise flow (the $\dot{\pi}=0$ isocline is still a straight line, but it is no longer horizontal). The trace condition for stability of the internal equilibrium $\left(\pi^{*}, p^{*}\right)$ is

$$
d \pi^{*}\left(1-\pi^{*}\right)+(1-\alpha) p^{*}\left(1-p^{*}\right)<0,
$$

while the heteroclinic orbit is attracting when

$$
\alpha^{2} \frac{c-d}{c+d}<1
$$




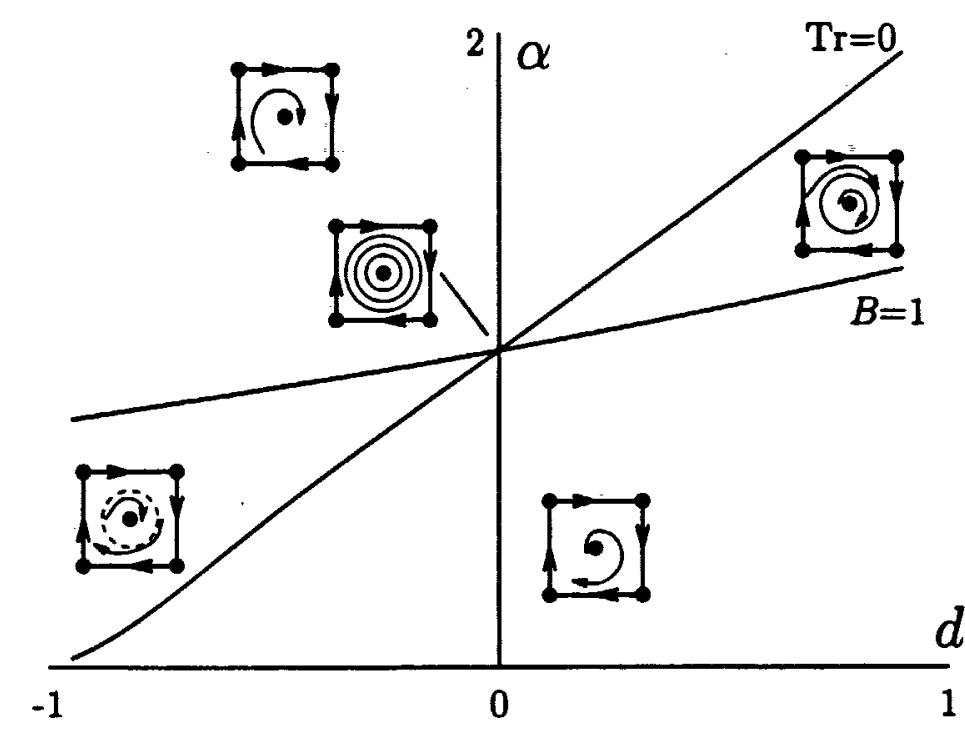

Figure 7: Bifurcation diagram for the symmetric model (26)-(27) describing a baculo virus. The parameter $c$ describes the component of viral selection induced by the host, and it is fixed at $c=4$. The parameter $d$ on the abscissa describes the frequency dependent fitness component of viral selection. The parameter $\alpha$ on the ordinate gives the strength of selection induced by a viral type against the host allele preferred by that viral type relative to the strength of selection against the other allele. The curve $\operatorname{tr} D=0$ shows where the internal equilibrium changes stability while $B=1$ indicates where the heteroclinic orbit $\gamma$ undergoes a global bifurcation. In the absence of frequency dependent selection $(d=0)$ the bifurcation is degenerate.

The two bifurcations now occur for different parameter values and the internal equilibrium undergoes a usual sub- or supercritical Hopf bifurcation, as indicated on the bifurcation diagram in Figure 7.

Therefore, direct viral interaction can break the degeneracy of the coevolution model (14)-(15). This may occur even if this effect is minor as suggested by field workers (Dwyer and Elkinton, 1993). Phenomena like superinfection may have a similar effect, and we conclude that the outcome of the coevolution will depend on such subtle aspects of the interaction. Thus, the occurrence of sustained coevolutionary cycles in models of phagebacterium systems (Levin et al., 1977) probably is due to phenomena that is not usually present in models of viral diseases.

\section{Conclusions}

Genetic variation with a small influence on the dynamical parameters of the interaction between a diploid host and a haploid pathogen leads to slow coevolution of the two species well described by a weak selection approximation. This process can end in various states. 
Selection may cause the pathogen or both the host and the pathogen to loose variation and end up monomorphic. Variants of a virus can only coexist when variation in the host is present. Selection may maintain stable polymorphism in both the host and the pathogen, but only if the host show overdominance in fitness at the stable equilibrium. Finally, selection may cause the host-pathogen system to cycle in a heteroclinic orbit where one of the species are nearly monomorphic while the other species goes through a trancient polymorphism. This cycling state is maintained by a steady flow of rare mutations in both host and pathogen.

Small changes in the dynamical parameters may change the end state from stable polymorphism in both species to a heteroclinic orbit when the dynamics is based on an SIR-model. In this sense we conclude that the evolutionary interaction between a diploid host and a haploid pathogen is degenerate in SIR-models. The abrupt change in dynamics eases as the genetic influence on the dynamical parameters becomes larger, but the range of parameters where limit cycles may exist is still very limited. We do not expect stable limit cycles in the genetic composition of the populations to be a prominent feature of the model unless the genetic variation has a significant impact on the dynamics.

The classical way of maintaining a two-allele polymorphism by selection is overdominance in survival. Selection in the host occurs through differential viability of the host genotypes, and the general result is that stable polymorphism occurs only when overdominance in fitness prevail at equilibrium. Thus, variation in the host is maintained when the heterozygote show more resistance against the disease than either of the two homozygotes. Situations where a stable genetic limit cycle that maintains variation by freqency-dependent selection without an obvious heterozygote advantage are virtually absent or extremely rare in models based on SIR-descriptions.

Variants of a virus cannot coexist unless the host varies, and the virus contributes to the maintenance of host polymorphism only in special circumstances, as we just saw. Therefore, variation is not expected to build up in the virus, and coexisting endemic vira has to be sufficiently different to overcome this version of the competitive exclusion principle.

A crusial assumption of the SIR-models is that the virus only has a existence of its own within the host or during the immediate transmission from one host to another. If this assumption is relaxed, then the degeneracy may be broken and non-trivial twospecies polymorphism result. We analysed the condition for non-degenerate behavior in $\mathrm{s}$ simple symmetric model, and there a rather pronounced level of qualitative interaction is required to produce stable genetic limit cycle for weak selection. However, this will have to be analysed in models of a wider scope before a general description is reached.

Competing equilibria occur in many situations in the SIR-based model. Stable twospecies polymorphic equilibria may coexist with monomorphic equilibria, one species polymorphic equilibria may compete with monomorphic equilibria with the other virus, and 
monomorphic equilibria may be stable simultaneously. Of particular interest is competing equilibria where different virus types and different host alleles are represented, because the existence of these equilibria allow two-species polymorphism in a collection of semi-isolated local populations, a metapopulation:- For instance, if two monomorphic equilibria which differ in both host and pathogen are stable simultaneously, then every isolated population will end up monomorphic. For other reasons, maybe historical, the populations may have fixed at different monomorphic equilibria, and so the metapopulation show a stable two-species polymorphic equilibria. Such equilibria, slightly modified, still exist for a low ammount of genetic migration between the local populations, and with migration even the local population will be at a stable two-species polymorphic equilibrium.

The maintenance by metapopulation effects of variation in virulence and resistance is interesting in its own right, and for the study of this phenomenon SIR-models are good and simple tools. SIR-models, however, are problematic for the study of host-parasite coevolution in more panmictic models.

\section{A Multiple time scales}

In this appendix we sketch how the motion along the quasi steady state

$$
\varpi(p, \pi)=\left(S, I, \pi, p_{S}, \hat{p}, \check{p}, F_{S}, \hat{F}, \check{F}\right)=\left(S^{*}, I^{*}, \pi, p, p, p, 0,0,0\right) .
$$

can be determined. Our first step will be to show that $\varpi(p, \pi)$ is a stable steady state when $\varepsilon=0$.

The system (6) is autonomous for $\varepsilon=0$, so by our assumptions $S$ and $I$ settle to an endemic equilibrium $\left(S^{*}, I^{*}\right)$. The variable $\pi$ is stationary from equation (7), and the system (8) contains a one-dimensional singularity $p_{S}=\hat{p}=\check{p}=p$. This manifold is attracting, i. e. $\left(p_{S}, \hat{p}, \check{p}\right) \rightarrow(p, p, p)$ for $t \rightarrow \infty$. To see this, study the variables $\xi_{1}=p_{S}-\hat{p}$ and $\xi_{2}=p_{S}-\check{p}$, and observe that

$$
\begin{aligned}
& \frac{d \xi_{1}}{d t}=-\left(\frac{b \pi I}{S}+\beta S\right) \xi_{1}-\frac{b(1-\pi) I}{S} \xi_{2} \\
& \frac{d \xi_{2}}{d t}=-\frac{b \pi I}{S} \xi_{1}-\left(\frac{b(1-\pi) I}{S}+\beta S\right) \xi_{2} .
\end{aligned}
$$

Obviously this system has a unique fixed point at $\left(\xi_{1}, \xi_{2}\right)=(0,0)$. Since the trace is negative and the determinant is positive, and both are bounded away from zero for sufficiently large $t$, we have $\xi_{1}(t), \xi_{2}(t) \rightarrow 0$ for $t \rightarrow \infty$ (Coddington and Levinson, 1955, p. 315).

Once (8) has reached $p_{S}=\hat{p}=\check{p}$, the deviations from Hardy-Weinberg proportions $F_{Q}$ goes to zero when $p_{Q} \neq 0,1$. At $p_{Q}=0,1$ the right hand side of (9) is discontinuous and we will need additional arguments. Andreasen and Christiansen (1993) show for a similar 
model that if $\bar{p} \neq 0,1, F_{Q} \rightarrow 0$ as $t \rightarrow \infty$ on the fast time scale so that $F_{Q}=0$ is also stable in situations where $a$ or $A$ are fixed. We will not pursue this question further, but simply conclude that (6)-(9) contains a 2-dimensional stable manifold $\varpi$ of fixed points parameterized by $(\pi, p)$. Therefore for $\varepsilon>0$ a short transience will bring the system close to the attracting manifold $\varpi$.

The dynamics of $\pi$ on $\varpi$ can be determined directly from (7) by evaluation of $\psi_{\pi}$ at $\varpi(p, \pi)$ and we get

$$
\frac{d \pi}{d t}=\frac{d \hat{I}}{d t} \frac{1}{I}-\pi \frac{d I}{d t} \frac{1}{I}=\varepsilon\left(\frac{\hat{\psi}_{I}}{I}-\pi \frac{\hat{\psi}_{I}+\bar{\psi}_{I}}{I}\right)=\varepsilon\left((1-\pi) \frac{\hat{\psi}_{I}}{I}-\pi \frac{\bar{\psi}_{I}}{I}\right) .
$$

Since $\hat{\psi}_{I}$ is to be evaluated at $\varpi$, we get $\hat{\psi}_{I}=\left\{\hat{\beta}_{i} S \hat{I} \mid p\right\}+\left\{\hat{\tau}_{i} \beta S \hat{I} \mid p\right\}-\left\{\mu_{i} \hat{I}+\hat{\nu}_{i} \hat{I} \mid p\right\}$ where $\left\{k_{i} \mid p\right\}=k_{1} p^{2}+k_{2} 2 p q+k_{3} q^{2}$. Simple algebra now gives

$$
\dot{\pi}=\varepsilon \pi(1-\pi)\left(S\left\{\hat{\beta}_{i}-\check{\beta}_{i} \mid \dot{p}\right\}+\beta S\left\{\hat{\tau}_{i}-\check{\tau}_{i} \mid p\right\}-\left\{\hat{\nu}_{i}-\check{\nu}_{i} \mid p\right\}\right) .
$$

The dynamics of $p$ on $\varpi$ can be determined by noting that according to (8) the variable

$$
\eta=\beta S p_{S}+\frac{b \pi I}{S} \hat{p}+\frac{b(1-\pi) \dot{I}}{S} \check{p}
$$

follows the equation

$$
\frac{d \eta}{d t}=\varepsilon\left(\beta S \psi_{p S}+\frac{b \pi I}{S} \hat{\psi}_{p}+\frac{b(1-\pi) I}{S} \check{\psi}_{p}\right) .
$$

Since on $\varpi$ we have $\eta=(\beta S+b I / S) p$, this observation may be used to determine the value of $\dot{p}=\dot{\eta} /(\beta S+b I / S)$ on $\varpi$. Evaluation of the $\psi$ s on $\varpi$ now yields.(10)-(11).

The choice of $\eta$ may be seen as a special case of the projection method of Beck et al. (1984), for further details on this we refer to Andreasen and Christiansen (1993).

\section{B Model with free living stages}

A coevolutionary version of the Anderson and May (1981) model with free living stages (23) is obtained as in Section 2:

$$
\begin{aligned}
& \frac{d S_{i}}{d t}=B_{i}-\left[\mu+\varepsilon \mu_{i}\right] S_{i}-\left[\beta+\varepsilon \hat{\beta}_{i}\right] S_{i} \hat{W}-\left[\beta+\varepsilon \check{\beta}_{i}\right] S_{i} \check{W}, \quad i=1,2,3 \\
& \frac{d \hat{I}_{i}}{d t}=\left[\beta+\varepsilon \hat{\beta}_{i}\right] S_{i} \hat{W}-\left[\mu+\varepsilon \mu_{i}\right] \hat{I}_{i}-\left[\nu+\varepsilon \hat{\nu}_{i}\right] \hat{I}_{i}, \quad i=1,2,3 \\
& \frac{d \check{I}_{i}}{d t}=\left[\beta+\varepsilon \check{\beta}_{i}\right] S_{i} \check{W}-\left[\mu+\varepsilon \mu_{i}\right] \check{I}_{i}-\left[\nu+\varepsilon \check{\nu}_{i}\right] \check{I}_{i}, \quad i=1,2,3,
\end{aligned}
$$




$$
\begin{aligned}
\frac{d \hat{W}}{d t}= & \sum_{j}\left[\lambda+\epsilon \hat{\lambda}_{j}\right] \hat{I}_{j}-\sum_{j}\left[\beta+\varepsilon \hat{\beta}_{S_{j}}\right] S_{j} \hat{W} \\
& \quad-\sum_{j}\left[\beta+\varepsilon \hat{\beta}_{v j}\right] \hat{I}_{j} \hat{W}-\sum_{j}\left[\beta+\varepsilon \hat{\beta}_{V j}\right] \check{I}_{j} \hat{W}-(\varrho+\varepsilon \hat{\varrho}) \hat{W}, \\
\frac{d \check{W}}{d t}= & \sum_{j}\left[\lambda+\epsilon \check{\lambda}_{j}\right] \check{I}_{j}-\sum_{j}\left[\beta+\varepsilon \check{\beta}_{S_{j}}\right] S_{j} \check{W} \\
& \quad-\sum_{j}\left[\beta+\varepsilon \check{\beta}_{v j}\right] \hat{I}_{j} \check{W}-\sum_{j}\left[\beta+\varepsilon \check{\beta}_{V j}\right] \check{I}_{j} \check{W}-(\varrho+\varepsilon \check{\varrho}) \check{W} .
\end{aligned}
$$

The detailed description of the interaction between larvae and free virus is discussed in Section 4. Transforming this 11 dimensional system into total abundance, gene frequency, and deviation from Hardy-Weinberg as in Section 2, we get

$$
\begin{aligned}
\frac{d S}{d t} & =b N-\mu S-\beta S I-\beta S \check{I}+\varepsilon \psi_{S} \\
\frac{d I}{d t} & =\beta S W-(\nu+\mu) I+\varepsilon \psi_{I} \\
\frac{d W}{d t} & =\lambda I-\varrho W-\beta(S+I) W+\varepsilon \psi_{W} \\
\frac{d \pi_{I}}{d t} & =\frac{\beta S W}{I}\left(\pi_{W}-\pi_{I}\right)+\varepsilon \psi_{\pi I} \\
\frac{d \pi_{W}}{d t} & =\frac{\lambda I}{W}\left(\pi_{I}-\pi_{W}\right)+\varepsilon \psi_{\pi W} \\
\frac{d p_{S}}{d t} & =b \frac{\pi I}{S}\left(\hat{p}_{I}-p_{S}\right)+b \frac{(1-\pi) I}{S}\left(\check{p}_{I}-p_{S}\right)+\varepsilon \psi_{p s} \\
\frac{d \hat{p}_{I}}{d t} & =\frac{\beta S W}{I}\left(p_{S}-\hat{p}_{I}\right)+\varepsilon \hat{\psi}_{p} \\
\frac{d \check{p}_{I}}{d t} & =\frac{\beta S W}{I}\left(p_{S}-\check{p}_{I}\right)+\varepsilon \check{\psi}_{p} \\
\frac{d F_{S}}{d t} & =b \frac{N}{S}\left(\frac{\left(p-p_{S}\right)^{2}}{p_{S} q_{S}}\left(1-F_{S}\right)-\frac{p q}{p_{S} q_{S}} F_{S}\right)+\varepsilon \psi_{F S} \\
\frac{d \hat{F}_{I}}{d t} & =\frac{\beta S W}{I}\left(\frac{\left(p_{S}-\hat{p}_{I}\right)^{2}}{\hat{p}_{I} \hat{q}}\left(1-\hat{F}_{I}\right)-\frac{p_{S} q_{S}}{\hat{p}_{I} \hat{q}}\left(\hat{F}_{I}-F_{S}\right)\right)+\varepsilon \hat{\psi}_{F}
\end{aligned}
$$




$$
\frac{d \check{F}_{I}}{d t}=\frac{\beta S W}{I}\left(\frac{\left(p_{S}-\check{p}_{I}\right)^{2}}{\check{p}_{I} \check{q}}\left(1-\check{F}_{I}\right)-\frac{p_{S} q_{S}}{\check{p}_{I} \check{q}}\left(\check{F}_{I}-F_{S}\right)\right)+\varepsilon \check{\psi}_{F}
$$

where $\pi_{I}$ and $\pi_{W}$ denote the frequency of virus type $v$ in the infected class and in the free living stage, respectively.

The model contains a stable two dimensional singularity, $\varpi(\pi, p)=$

$$
\left\{\left(S, I, W, \pi_{I}, \pi_{W}, p_{S}, \hat{p}_{I}, \check{p}_{I}, F_{S}, \hat{F}_{I}, \check{F}_{I}\right)=\left(S^{*}, I^{*}, W^{*}, \pi, \pi, p, p, p, 0,0,0\right) \mid 0 \leq \pi \leq 1,0 \leq p \leq 1\right\},
$$

The variables $\eta_{\pi}$ and $\eta_{p}$, where

$$
\begin{aligned}
& \eta_{\pi}=\frac{\lambda I}{W} \pi_{I}+\frac{\beta S W}{I} \pi_{W} \\
& \eta_{p}=\frac{\beta S W}{I} p_{S}+\frac{\pi b I}{S} \hat{p}_{I}+\frac{(1-\pi) b I}{S} \check{p}_{I}
\end{aligned}
$$

are slow variables so that

$$
\begin{aligned}
& \frac{d \pi}{d t}=\varepsilon\left(\frac{\lambda I}{W} \psi_{\pi I}+\frac{\beta S W}{I} \psi_{\pi W}\right) /\left(\frac{\lambda I}{W}+\frac{\beta S W}{I}\right) \\
& \frac{d p}{d t}=\varepsilon\left(\frac{\beta S W}{I} \psi_{p S}+\frac{\pi b I}{S} \hat{\psi}_{p}+\frac{(1-\pi) b I}{S} \bar{\psi}_{p}\right) /\left(\frac{\beta S W}{I}+\frac{b I}{S}\right) .
\end{aligned}
$$

The coefficients $\psi$ need only be evaluated on the slow manifold, and we find

$$
\begin{aligned}
\psi_{\pi I}= & (1-\pi) \pi\left\{\left(\hat{\beta}_{i}-\check{\beta}_{i}\right) S W / I-\left(\hat{\nu}_{i}-\check{\nu}_{i}\right) \mid p\right\} \\
\psi_{\pi W}= & (1-\pi) \pi\left\{\left(\hat{\lambda}_{i}-\check{\lambda}_{i}\right) I / W-\left(\hat{\beta}_{i}-\check{\beta}_{i}\right) S-\left(\hat{\beta}_{v i}-\check{\beta}_{v i}\right) \pi I-\left(\hat{\beta}_{V i}-\check{\beta}_{V i}\right)(1-\pi) I \mid p\right\} \\
& \quad-(1-\pi) \pi(\hat{\varrho}-\check{\varrho}) \\
\psi_{p s}= & -p(1-p)\left\langle\mu_{i}+\hat{\beta}_{i} W \pi+\check{\beta}_{i} W(1-\pi) \mid p\right\rangle . \\
\hat{\psi}_{p}= & p(1-p)\left\langle\hat{\beta}_{i} W S / I-\hat{\nu}_{i}-\mu_{i} \mid p\right\rangle \\
\dot{\psi}_{p}= & p(1-p)\left\langle\check{\beta}_{i} W S / I-\check{\nu}_{i}-\mu_{i} \mid p\right\rangle .
\end{aligned}
$$

The weak selection model (24)-(25) now follows.

\section{References}

Anderson, R. M. and May, R. M. (1979). Population biology of infectious diseases: Part I. Nature (London), 280, 361-367.

Anderson, R. M. and May, R. M. (1981). The population dynamics of macroparasites and their invertebrate hosts. Phil. Trans. Roy. Soc. London, B291, 451-524. 
Anderson, R. M. and May, R. M. (1982). Coevolution of hosts and parasites. Parasitology, $85,411-426$.

Andreasen, V. and Christiansen, F. B. (1993). Disease-induced natural seceltion in a diploid host. Theor. Pop. Biol., 44, 261-298.

Babour, A. D. (1978). Macdonald's model and the transmission of bilharzia. Trans. Roy. Soc. Tropical Med. and Hygiene, 72, 6-15.

Beck, K. (1984). Coevolution: Mathematical analysis of host-parasite interactions. $J$. Math. Biol., 19, 63-77.

Beck, K., Keener, J. P., and Ricciardi, P. (1984). The effect of epidemics on genetic evolution. J. Math. Biol., 19, 79-94.

Blythe, S. and Castillo-Chavez, C. (1989). Like-with-like preference and sexual mixing models. Math. Biosci., 96, 221-238.

Bremermann, H. and Thieme, H. (1989). A competitive exclusion principle for pathogen virulence. J. Math. Biol., 27, 179-190.

Clarke, B. C. (1979). The evolution of genetic diversity. Proc. R. Soc. Lond., B246, 453-474.

Coddington, E. A. and Levinson, N. (1955). Theory of Ordinary Differential Equations. McGraw-Hill, New York.

Dietz, K. (1975). Transmission and control of arbovirus diseases. In D. Ludwig and K. L. Cooke (Eds.), Epidemiology (pp. 104-121). Society for Industrial and Applied Mathematics, Philadelphia.

Dwyer, G. and Elkinton, J. E. (1993). Using simple models to predict virus epizootics in gypsy moth populations. J. Anim. Ecol., 62, 1-11.

Ewald, P. W. (1983). Host-parasite relations, vectors, and the evolution of disease severity. Ann. Rev. Ecol. Syst., 14, 465-485.

Fisher, R. A. (1930). The Genetical Theory of Natural Selection. Claredon Press, Oxford.

Fraenkel-Conrat, H., Kimball, P. C., and Levy, J. A. (1988). Virology. Prentice Hall, Englewood Cliffs, N. J.

Gillespie, J. H. (1975). Natural selection for resistance to epidemics. Ecology, 56, 493-495.

Guckenheimer, J. and Holmes, P. (1983). Nonlinear Oscillations, Dynamical Systems, and Bifurcations of Vector Fields. Springer-Verlag, Berlin, Heidelberg, New York. 
Haldane, J. B. S. (1949). Disease and evolution. Ricera Scient. (suppl.), 19, 68-76.

Hethcote, H. W. (1974). Asymptotic behavior and stability of epidemic models. In P. van der Driessche (Ed.), Mathematical Problems in Biology (pp. 83-92). SpringerVerlag, Berlin, Heidelberg, New York.

Hethcote, H. W. and Yorke, J. A. (1981). Gonorrhea Transmission Dynamics and Control. Springer-Verlag, Berlin, Heidelberg, New York.

Hoppensteadt, F. (1966). Singular perturbations on the infinite interval. Trans. Amer. Math. Soc., 123, 521-535.

Jacquez, J., Simon, C., Koopman, J., Sattenspiel, L., and Perry, T. (1988). Modelling and analyzing HIV transmission: the effect of contact patterns. Math. Biosci., 92, 119-199.

Jayakar, S. D. (1970). A mathematical model for interaction between gene frequencies in a parasite and its host. Theor. Pop. Biol., 1, 140-164.

Kemper, J. T. (1982). The evolutionary effect of endemic infectious disease: Continuous models for an invariant pathogen. J. Math. Biol., 15, 65-77.

Kimura, M. (1958). On the change of population fitness by natural selection. Heredity, $12,145-167$.

Knolle, H. (1989). Host density and the evolution of parasite virulence. J. Theor. Biol., 136, 199-207.

Levin, B. (1988). Frequency-dependent selection in bacterial populations. Phil. Trans. $R$. Soc. Lond., B319, 459-472.

Levin, B. and Lenski, R. E. (1983). Coevolution in bacteria and their viruses and Plasmids. In D. J. Futuyma and M. Slatkin (Eds.), Coevolution (pp. 21-65). Sinauer Associates, Sunderland, Massachusetts.

Levin, B., Stuart, F. M., and Chao, L. (1977). Resource limited growth, competition and predation: A model and some experimental studies with bacteria and bacteriophage. Amer. Natur., 111, 3-24.

Levin, S. A. (1983). Some approaches to the modelling of coevolutionary interactions. In M. Nitecki (Ed.), Coevolution (pp. 21-65). University of Chicago Press, Chigaco.

Levin, S. A. and Pimentel, D. (1981). Selection for intermediate rates of increase in parasite-host systems. Amer. Nat., 117, 308-315. 
Levin, S. A. and Udovic, J. D. (1977). A mathematical model of coevolving populations. Amer. Nat., 111, 657-675.

Lewis, J. W. (1981). On the coevolution of pathogen and host: I. General theory of discrete time coevolution. J. theor. Biol., 93, 927-951.

Liu, W., Levin, S. A., and Iwasa, Y. (1986). Influence of non-linear incidence rates upon the behavior of SIRS epidemiological models. J. Math. Biol., 23, 187-204.

Longini, I. M. (1983). Models of epidemics and endemicity in genetically variable host populations. J. Math. Biol., 17, 289-304.

May, R. M. and Anderson, R. M. (1983). Epidemiology and genetics in the coevolution of parasites and hosts. Proc. R. Soc. Lond., B219, 281-313.

Nagylaki, T. (1976). The evolution of one- and two-locus systems. Genetics, 83, 583-600.

Norton, H. T. J. (1928). Natural selection and Mendelian variation. Proc. Lond. Math. Soc. (Ser. 2), 28, 1-45.

Nowak, M. and May, R. M. (1994). Superinfection and the evolution of parasite virulence. Proc. Roy. Soc. London B, 255, 81-89.

Pugliese, A. (1990). Population models for diseases with no recovery. J. Math. Biol., 28, 65-82.

Sasaki, A. and Iwasa, Y. (1991). Optimal growth schedule of pathogens within a host: Switching between lytic and latent cycles. Theor. Pop. Biol., 39, 201-239.

Segel, L. A. (1988). On the validity of the steady state asssumption of enzyme kinetics. Bull. Math. Biol., 50, 579-593.

Stuart, F. M. and Levin, B. (1984). The population biology of bacterial viruses: Why be temperate. Theor. Pop. Biol., 26, 93-117.

Wright, S. (1955). Classification of the factors of evolution. Cold Spring Harbor Symp. Quant. Biol., 20, 16-24D.

Yu, P. (1972). Some host-parasite genetic interaction models. Theor. Popul. Biol., 3, 347-357. 

$236 a / 93$ INTRODUKTION TIL KVANTE HALL EFFEKTEN

ar: An le Boisen, Peter Boggild

Vejleder: Peder Voetmann Christiansen Erland Brun Hansen

236 b/93 STROMSSAMMENBRUD AF KVANTE HALL EFFEKTEN

af: Anja Boisen. Peter Besgild

Vejleder: Peder Voetmann Christiansen Erland Brun Hansen

237/93 The wedderburn principal theorem and Shukla cohomology

af: Lars Kadison

$238 / 93$ SEMIOTIK OG SYSTEMEGENSKABER

Vektorbind og tensorer

af: Peder Voetmann Christiansen

$239 / 93$ Valgsystemer - Modelbygning og analyse Matematik 2 . modul

af: Charlotte Gjerfild, Jane Hansen, Maria Hermannsson, Allan Jorgensen.

Ragna Clauson-kas, Poul Lützen

Vejleder: Mogens Niso

240/93 Patologiske eksempler.

Om sere matematiske fisks betydning for den matematiske udvikling

of: Ciaus Dreby, Jorn Skov Hansen, Runa Ulsoe Johansen. Peter Mejbom, Johannes Kristorfer Nielsen

Vejleder: Mogens Niss

241,93 FOTOVOLTAISK STATUSNOTAT 1

af: Bent Sorensen

242,93 Brovealigeholdelse - bevar mig vel

Analyse af Vejdirektoratets model for optimering af broreparationer

af: Linca Kyndlev, Kare Fundal, Kamma

Tulinius, Ivar zeck

Vejleder: Jesper Larsen

243/93 TANKEEKSPERIMENTER I FYSIKKEN

Et l.modul fysikprojekt

af: Karen Birkelund, Stine Sofia Korremann

Vejleder: Dorthe Posselt

244/93 RADONTRANSFORMATIONEN Oq dens anvendelse i CT-scanning

Projektrapport

af: Trine Andreasen, Tine Gulaager Christiansen, Nina Skov Hansen og Christine Iversen

Vejledere: Gestur olafsson og Jesper Larsen

$245 a+b$

193 Time-Of-Flight málinger på krystallinske

halviedere

Specialerapport

af: Linda Szkotak Jensen og Lise Odgaard Gade

Vejledere: Petr Viscor og Niels Boye olsen

$246 / 93$ HVERDAGSVIDEN OG MATEMATIK

- LRREPROCESSER I SKOLEN

af: Lena Lindenskov, Statens Humanistiske

Forskningsrad, RUC, IMFUFA
247,93 UNIVERSAL LOW TEMPERATURE AC CONDUCTIVITY OF MACROSCOPICALLY DISORDERED NON-METAIS

by: Jeppe C. Dyre

$248 / 93$ DIRAC OPERATORS AND MANIFOLDS WITH BOUNDARY

by: B. Booss-Bavnbek, R.P.Wojciechowsk 2

$249 / 93$ Perspectives on Teichmiller and the zahresbericht Addendum to Schappacher. Scholz, et al.

by: B. Booss-Bavnbek

With comments by W.Abikoff, I.Ahlfors. J.Cerf, P.J.Davis, W.Fuchs, F.P.Gardiner. J.Jost, J.-P. Kahane, R.Lohan, L.Lorch, I.Radkau and T.Söderquist

250/93 EULER OC BOL2ANO - NATEMATISK ARALYSE SET I EJ VIDENSKABSTEORETTSK PERSPEKTIV

Projektrapport af: Anja Juul, tone Nichelser., Tomas bojgknd Jensen

Vejleder: Stis Andur Pedersen

$851 \mid 83$ Cenotypic Proportions in Bybrid zones

by: Fraddy Bugge Crristiorsen, Piggo Andreasen and Bbbe True Pouleen

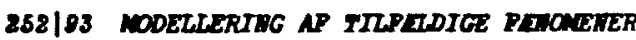

Frojektrapport af: Birthe Prits, Lisbeth Belapaar krist ine Chor Lot te Jakobsen, Marina Nobek

Johorressen, Lotte Ludvigsen, Nette Base Bielsen

353/93 ruglepaking

Teori og model

of: Lioe Arlath, Bore Padal, Dils knuse

Veflader: Mogens Wise

251/83 Regreseionsanalyse

Materiale til ot otatiotiklaores

af: Jorgen Loreen

255/93 TID \& BETHEET UAPBINGTGBED

af: Peter dommoēs

256/93 Deterwination of the Prequency Dependent Bulk Mbchlus of Liquids Using a Pienoelectrie Spherical shell (Proprint)

by: I. Chrietensen and W.B.oteen

$867 / 03$ Modellering af diopersion i piesontektriake kerowikker

af: Pernille Fostgarad, Jewik Rasmussen, Croistina spocht, Mikte catargoud

Vejleder: Tage Christensen

858/98 Suphomale boreumatariale til

Hinge etruktures fro algabre og enalyee"

af: Nogens Bron Biefelt

250193 STUDTES OP AC BOPPING CODUCTTOA AT LOW IUPERATURES

by: Jepe C. Dyre

260193 PARTITIONED MTIPOLOS AID MWARIATSS IN DIRTSIONS 8, 3, AND

by: B. Boose-Baumbe, X.P. Hojeicchoweki 


\section{1/93 OPGAVESAMLING}

Bredde-kursus i Fysik

Eksamensopgaver fra $1976-93$

262/93 Separability and the Jones Polynomial

by: Lars Kadison

263/93 Supplezende kursusmateriale til "Lineare strukturer fra algebra oa analyse" II

af: Mogens Brun Heefelt

$264: 93$ FOTOVOLTAISK STATUSNOTAT 2 af: Bent Sorensen

265/94 SPHERICAL FUACTIONS ON ORDERED SMMETRIC SPACES

To sigurdur Belgason on his sixtyfifth birthday

by: Jacques Faraut, Joachis Hilgert and Gestur Olafison

266/94 Kommensurabilitets-oscillationer i laterale supergitre Fysikspeciale af: Anja Boisen, Peter Boggild, Karen Birkelund

Vejledere: Rafael. Taboryski, Poul Erik Lindelof, Peder Voetmann Christiansen

267/94 Kom til kort med matematik på Eksperimentarium - Et forslag til en opstilling

af: Charlotte Gjerrild, Jane Hansen Vejleder: Bernhelm Booss-Bavnbek

268/94 Iife is like a sewer ...

Et projekt om modellering af aorta via en model for stromning $i$ kloakror

af: Anders Marcussen, Anne C. Nilsson, Lone Michelsen, Per M. Hansen

Vejleder: Jesper Larsen

269/94 Dimensionsanalyse en introduktion metaprojekt, fysik

af: Tine Guldager Christiansen,

Ken Andersen, Nikolaj Hermann,

Jannik' Rasmussen

Vejleder: Jens Hojgaard Jensen

270/94 THE IMAGE OF THE ENVELOPING ALGEBRA AND IRREDUCIBILITY OF INDUCED REPRESENTATIONS OF EXPONENTIAL LIE GROUPS

by: Jacob zacobsen

271/94 Matematikken.i Fysikken.

Opaaget eller opfundet

NAT-BAS-projekt

vejleder: Jens Hojgaard Jensen
272/94 Tradition og fornyelse

Det praktiske elevarbejde 1 ovmnasiets fysikundervisning, 1907-1988

af: Kristian Hoppe oB Jeppe Guldager

Vejledning: Karin Beyer og Nils Hybel

273/94 Model for kort- og mellendistancelob Verifikation af model

af: Lise Fabricius Christensen, Helle Pilemann, Bettina Sorensen

Vejleder: Mette Olufien

274/94 MODEL 10 - en matematisk model af intravenose ankstetikas ramakokinetik

3. modul matematik, forár 1994

ar: Trine Andreasen, Bjorn Christensen, Christine Green, Anja Skjoldborg Hansen. Lisbeth Helmgaard

Vejledere: Viggo Andreasen \& Jesper Larsen

275/94 Perspectives on Ieichmiller and the Jahresbericht 2nd Edition

by: Bernhelm Booss-Bavnbek

276/94 Dispersionsmodellering

Projektrapport 1. modul

af: Gitte Andersen, Rehannah Borup, Lisbeth Fris, Per Gregersen, Kristina Vejro

Vejleder: Bernhelm Booss-Bavnbek.

277/94 PROJEKTARBEJDSPEDAGOGIK - Om tre tolkninger af problemorienteret projektarbejde

af: Claus Flensted Behrens, Frederik Voetmann Christiansen, Jorn Skov Hansen, Thomas Thingstrup

Vejleder: Jens hojgaard Jensen

278/94 The Models Underlying the Anaesthesia Simulator Sophus

by: Mette Olufsen(Math-Tech), Finn Nielsen (RISo National Laboratory). Per Foge Jensen (Herlev University hospital), Stig Andur Pedersen (Roskilde University)

279/94 Description of a method of measuring the shear modulus of supercooled liquids and a comparison of their thermal and mechanlcal response functions.

af: Tage Christensen

280/94 A Course in Projective Geometry by Lars Kadison and Matthias T. Kromann

281/94 Modellering af Det Cardiovaskulere System med Neural Pulskontrol

Projektrapport udarbejdet af:

Steran Frello, Runa Ulsoe Johansen, Michael Poul Curt Mansen, Kleus Dahl Jensen Vejleder: Viggo Andreasen 